Article

\title{
A General Theorem on the Stability of a Class of Functional Equations Including Quartic- Cubic-Quadratic-Additive Equations
}

\author{
Yang-Hi Lee ${ }^{1}$ and Soon-Mo Jung ${ }^{2, * D}$ \\ 1 Department of Mathematics Education, Gongju National University of Education, Gongju 32553, Korea; \\ yanghi2@hanmail.net \\ 2 Mathematics Section, College of Science and Technology, Hongik University, Sejong 30016, Korea \\ * Correspondence: smjung@hongik.ac.kr; Tel.: +82-44-860-2584
}

Received: 21 October 2018; Accepted: 21 November 2018; Published: 26 November 2018

Abstract: We prove general stability theorems for $n$-dimensional quartic-cubic-quadratic-additive type functional equations of the form $\sum_{i=1}^{\ell} c_{i} f\left(a_{i 1} x_{1}+a_{i 2} x_{2}+\cdots+a_{i n} x_{n}\right)=0$ by applying the direct method. These stability theorems can save us the trouble of proving the stability of relevant solutions repeatedly appearing in the stability problems for various functional equations.

Keywords: generalized Hyers-Ulam stability; functional equation; $n$-dimensional quartic-cubicquadratic-additive type functional equation; direct method

MSC: 39B82, 39B52

\section{Introduction}

The stability problem for functional equations or differential equations began with the well known question of Ulam [1]:

Let $G_{1}$ and $G_{2}$ be a group and a metric group with a metric $d(\cdot, \cdot)$, respectively. Given $\varepsilon>0$, does there exist a $\delta>0$ such that if a function $h: G_{1} \rightarrow G_{2}$ satisfies the inequality $d(h(x y), h(x) h(y))<\delta$ for all $x, y \in G_{1}$, then there exists a homomorphism $H: G_{1} \rightarrow G_{2}$ with $d(h(x), H(x))<\varepsilon$ for all $x \in G_{1}$ ?

In short, the Ulam's question states as follows: Under what conditions does there exist an additive function near an approximately additive function? (For more details about the historical background, see [1-5].)

In 1941, Hyers [6] gave a partial solution to the question of Ulam under the assumption that relevant functions are defined on Banach spaces. Indeed, Hyers' (modified) theorem states that the following statement is true for all $\varepsilon \geq 0$ : If a function $f$ satisfies the inequality $\| f(x+y)-f(x)-$ $f(y) \| \leq \varepsilon$ for all $x$, then there exists an exact additive function $F$ and a function $K:[0, \infty) \rightarrow[0, \infty)$ such that $\|f(x)-F(x)\| \leq K(\varepsilon)$ for all $x$ and $\lim _{\varepsilon \rightarrow 0} K(\varepsilon)=0$. In this case, the Cauchy additive functional equation, $f(x+y)=f(x)+f(y)$, is said to have (or satisfy) the Hyers-Ulam stability.

When Hyers' theorem is true even if we replace $\varepsilon$ and $K(\varepsilon)$ by $\varphi(x)$ and $\Phi(x)$, where $\varphi$ and $\Phi$ are functions not depending on $f$ and $F$ explicitly, the corresponding equation is said to have (or satisfy) the generalized Hyers-Ulam stability. These terminologies will also be applied for other functional equations.

Since Hyers' paper, a number of mathematicians have extensively investigated the stability problems for several functional equations (see for example $[2-5,7-15]$ and the references therein). 
Given abelian groups $G_{1}$ and $G_{2}$ and any mapping $f: G_{1} \rightarrow G_{2}$, we will set

$$
\begin{aligned}
A f(x, y) & :=f(x+y)-f(x)-f(y) \\
Q f(x, y) & :=f(x+y)+f(x-y)-2 f(x)-2 f(y) \\
C f(x, y) & :=f(2 x+y)-3 f(x+y)+3 f(y)-f(-x+y)-6 f(x) \\
Q^{\prime} f(x, y) & :=f(2 x+y)-4 f(x+y)+6 f(y)-4 f(-x+y)+f(-2 x+y)-24 f(x)
\end{aligned}
$$

for all $x, y \in G_{1}$. In connection with these notations, a mapping $f: G_{1} \rightarrow G_{2}$ is said to be a quartic mapping, a cubic mapping, a quadratic mapping, or an additive mapping provided $f$ satisfies the functional equation $Q^{\prime} f(x, y)=0, C f(x, y)=0, Q f(x, y)=0$ or $A f(x, y)=0$ for all $x, y \in G_{1}$, respectively. Those functional equations seem to be familiar to us because we can easily find out examples for the existence of their solutions. For example, the mapping $f: \mathbb{R} \rightarrow \mathbb{R}$ given by $f(x)=a x^{4}$, $a x^{3}, a x^{2}$ or $a x$ is a solution of $Q^{\prime} f(x, y)=0, C f(x, y)=0, Q f(x, y)=0$, or $A f(x, y)=0$, respectively.

If a mapping $f: G_{1} \rightarrow G_{2}$ can be represented by the sum of a quartic mapping, a cubic mapping, a quadratic mapping, and an additive mapping, then we call $f$ a quartic-cubic-quadratic-additive mapping and vice versa. For example, the mapping $f: \mathbb{R} \rightarrow \mathbb{R}$ given by $f(x)=a x^{4}+$ $b x^{3}+c x^{2}+d x$ is a quartic-cubic-quadratic-additive mapping. A functional equation is said to be a quartic-cubic-quadratic-additive functional equation provided the set of all of its solutions is the same as the set of all quartic-cubic-quadratic-additive mappings.

Throughout this paper, we assume that $V$ and $W$ are real vector spaces, $X$ a real normed space, and that $Y$ is a real Banach space if there is no specification. Let $\mathbb{N}_{0}$ be the set of all nonnegative integers, $\mathbb{R}$ the set of all real numbers, and let $\mathbb{Q}$ denote the set of all rational numbers.

In this paper, we prove some general stability theorems that can be easily applied to the (generalized) Hyers-Ulam stability of a large class of functional equations of the form

$$
D f\left(x_{1}, x_{2}, \ldots, x_{n}\right)=0,
$$

which includes quartic-cubic-quadratic-additive functional equations. From now on, for any given mapping $f: V \rightarrow W$, we define $D f: V^{n} \rightarrow W$ by

$$
D f\left(x_{1}, x_{2}, \ldots, x_{n}\right):=\sum_{i=1}^{\ell} c_{i} f\left(a_{i 1} x_{1}+a_{i 2} x_{2}+\cdots+a_{i n} x_{n}\right)
$$

for all $x_{1}, x_{2}, \ldots, x_{n} \in V$, where $\ell$ and $n$ are fixed integers larger than 1 , and $c_{i}, a_{i j}$ are fixed real constants throughout this paper. The direct method, which is the most powerful one in studying the stability problems of functional equations, is mainly applied to the proof of main theorems of this paper and the paper [10], while another method known as the "fixed point approach" was applied to the proof of the main theorem of [11].

Until now, we have followed out a routine and monotonous procedure for studying the stability problems of the quartic-cubic-quadratic-additive functional equations. However, the stability theorems of this paper can save us the trouble of proving the stability of relevant solutions repeatedly appearing in the stability problems for various functional equations (see [16-20]). 


\section{Preliminaries}

Throughout this section, let $V$ and $W$ be real vector spaces and let $Y$ be a real Banach space. Given a mapping $f: V \rightarrow W$ and a real number $a$, we will use the following notations:

$$
\begin{array}{lll}
f_{1}(x):=\frac{a^{3} f_{o}(x)-f_{o}(a x)}{a^{3}-a}, & f_{2}(x):=\frac{a^{4} f_{e}(x)-f_{e}(a x)}{a^{4}-a^{2}}, \\
f_{3}(x):=\frac{f_{o}(a x)-a f_{o}(x)}{a^{3}-a}, & f_{4}(x):=\frac{f_{e}(a x)-a^{2} f_{e}(x)}{a^{4}-a^{2}}, \\
f_{o}(x):=\frac{f(x)-f(-x)}{2}, & f_{e}(x):=\frac{f(x)+f(-x)}{2}
\end{array}
$$

for all $x \in V$. We will now introduce some lemmas from [21] in the following Corollaries 1-4.

Lemma 1 ([21] (Corollary 3.1)). Given a real constant $k>1$, assume that a function $\phi: V \backslash\{0\} \rightarrow[0, \infty)$ satisfies either the condition

$$
\left.\Phi(x):=\sum_{i=0}^{\infty} \frac{1}{k^{i}} \phi\left(k^{i} x\right)<\infty \quad \text { for all } x \in V \backslash\{0\}\right)
$$

or

$$
\left.\Phi(x):=\sum_{i=0}^{\infty} k^{4 i} \phi\left(\frac{x}{k^{i}}\right)<\infty \quad \text { for all } x \in V \backslash\{0\}\right)
$$

Furthermore, suppose $f: V \rightarrow Y$ is an arbitrary mapping. If a mapping $F: V \rightarrow Y$ satisfies the inequality

$$
\|f(x)-F(x)\| \leq \Phi(x)
$$

for all $x \in V \backslash\{0\}$ as well as the equalities

$$
\begin{array}{ll}
F_{1}(k x)=k F_{1}(x), & F_{2}(k x)=k^{2} F_{2}(x), \\
F_{3}(k x)=k^{3} F_{3}(x), & F_{4}(k x)=k^{4} F_{4}(x)
\end{array}
$$

for all $x \in V$, then $F$ is the unique mapping with the properties of (5) and (6).

Lemma 2 ([21] (Corollary 3.2)). Given a real number $k>1$, assume that the functions $\phi, \psi: V \backslash\{0\} \rightarrow[0, \infty)$ satisfy each of the following conditions

$$
\begin{array}{cc}
\sum_{i=0}^{\infty} k^{i} \psi\left(\frac{x}{k^{i}}\right)<\infty, & \sum_{i=0}^{\infty} \frac{1}{k^{2 i}} \phi\left(k^{i} x\right)<\infty, \\
\tilde{\Phi}(x):=\sum_{i=0}^{\infty} k^{i} \phi\left(\frac{x}{k^{i}}\right)<\infty, & \tilde{\Psi}(x):=\sum_{i=0}^{\infty} \frac{1}{k^{2 i}} \psi\left(k^{i} x\right)<\infty
\end{array}
$$

for all $x \in V \backslash\{0\}$, and $f: V \rightarrow Y$ is an arbitrary mapping. If a mapping $F: V \rightarrow Y$ satisfies the inequality

$$
\|f(x)-F(x)\| \leq \tilde{\Phi}(x)+\tilde{\Psi}(x)
$$

for all $x \in V \backslash\{0\}$ as well as the equalities in Expression (6) for all $x \in V$, then $F$ is the unique mapping satisfying equalities in (6) for all $x \in V$ and the inequality (7) for all $x \in V \backslash\{0\}$. 
Lemma 3 ([21] (Corollary 3.3)). Given a real number $k>1$, assume that the functions $\phi, \psi: V \backslash\{0\} \rightarrow[0, \infty)$ satisfy each of the following conditions

$$
\begin{array}{cc}
\sum_{i=0}^{\infty} k^{2 i} \psi\left(\frac{x}{k^{i}}\right)<\infty, & \sum_{i=0}^{\infty} \frac{1}{k^{3 i}} \phi\left(k^{i} x\right)<\infty, \\
\tilde{\Phi}(x):=\sum_{i=0}^{\infty} k^{2 i} \phi\left(\frac{x}{k^{i}}\right)<\infty, & \tilde{\Psi}(x):=\sum_{i=0}^{\infty} \frac{1}{k^{3 i}} \psi\left(k^{i} x\right)<\infty
\end{array}
$$

for all $x \in V \backslash\{0\}$, and $f: V \rightarrow Y$ is an arbitrary mapping. If a mapping $F: V \rightarrow Y$ satisfies the inequality (7) for all $x \in V \backslash\{0\}$ as well as the equalities in (6) for all $x \in V$, then $F$ is the unique mapping satisfying the equalities in (6) for all $x \in V$ and the inequality (7) for all $x \in V \backslash\{0\}$.

Lemma 4 ([21] (Corollary 3.4)). Assume that $k>1$ is a real number and the functions $\phi, \psi: V \backslash\{0\} \rightarrow[0, \infty)$ satisfy each of the following conditions

$$
\begin{array}{cc}
\sum_{i=0}^{\infty} k^{3 i} \psi\left(\frac{x}{k^{i}}\right)<\infty, & \sum_{i=0}^{\infty} \frac{1}{k^{4 i}} \phi\left(k^{i} x\right)<\infty, \\
\tilde{\Phi}(x):=\sum_{i=0}^{\infty} k^{3 i} \phi\left(\frac{x}{k^{i}}\right)<\infty, & \tilde{\Psi}(x):=\sum_{i=0}^{\infty} \frac{1}{k^{4 i}} \psi\left(k^{i} x\right)<\infty
\end{array}
$$

for all $x \in V \backslash\{0\}$. In addition, suppose $f: V \rightarrow Y$ is an arbitrary mapping. If a mapping $F: V \rightarrow Y$ satisfies the inequality (7) for all $x \in V \backslash\{0\}$ as well as the equalities in (6) for all $x \in V$, then $F$ is the unique mapping satisfying the equalities in (6) for all $x \in V$ and the inequality (7) for all $x \in V \backslash\{0\}$.

\section{Main Results}

In this section, we assume that $V$ is a real vector space and $Y$ is a real Banach space if there is no specification. In the following theorems, we prove that there exists only one exact solution near every approximate solution to $D f\left(x_{1}, x_{2}, \ldots, x_{n}\right)=0$.

Theorem 1. Given a real constant a with $|a|>1$, assume that the functions $\mu, v: V \backslash\{0\} \rightarrow[0, \infty)$ satisfy the conditions

$$
\sum_{i=0}^{\infty} \frac{\mu\left(a^{i} x\right)}{|a|^{i}}<\infty \text { and } \sum_{i=0}^{\infty} \frac{v\left(a^{i} x\right)}{|a|^{i}}<\infty
$$

for all $x \in V \backslash\{0\}$ and a function $\varphi:(V \backslash\{0\})^{n} \rightarrow[0, \infty)$ satisfies the condition

$$
\sum_{i=0}^{\infty} \frac{\varphi\left(a^{i} x_{1}, a^{i} x_{2}, \ldots, a^{i} x_{n}\right)}{|a|^{i}}<\infty
$$

for all $x_{1}, x_{2}, \ldots, x_{n} \in V \backslash\{0\}$. If a mapping $f: V \rightarrow Y$ satisfies $f(0)=0$,

$$
\begin{aligned}
& \left\|f_{e}\left(a^{2} x\right)-\left(a^{2}+a^{4}\right) f_{e}(a x)+a^{6} f_{e}(x)\right\| \leq \mu(x), \\
& \left\|f_{o}\left(a^{2} x\right)-\left(a+a^{3}\right) f_{o}(a x)+a^{4} f_{o}(x)\right\| \leq v(x)
\end{aligned}
$$

for all $x \in V \backslash\{0\}$, and, if $f$ moreover satisfies the inequality

$$
\left\|D f\left(x_{1}, x_{2}, \ldots, x_{n}\right)\right\| \leq \varphi\left(x_{1}, x_{2}, \ldots, x_{n}\right)
$$


for all $x_{1}, x_{2}, \ldots, x_{n} \in V \backslash\{0\}$, then there exists a unique mapping $F: V \rightarrow Y$ such that

$$
D F\left(x_{1}, x_{2}, \ldots, x_{n}\right)=0
$$

for all $x_{1}, x_{2}, \ldots, x_{n} \in V \backslash\{0\}$, and

$$
\begin{array}{ll}
F_{1}(a x)=a F_{1}(x), & F_{2}(a x)=a^{2} F_{2}(x), \\
F_{3}(a x)=a^{3} F_{3}(x), & F_{4}(a x)=a^{4} F_{4}(x)
\end{array}
$$

for all $x \in V$, and such that

$$
\|f(x)-F(x)\| \leq \sum_{i=0}^{\infty}\left(\frac{\left|a^{2 i+2}-1\right| \mu\left(a^{i} x\right)}{\left|a^{4}-a^{2}\right||a|^{4 i+4}}+\frac{\left|a^{2 i+2}-1\right| v\left(a^{i} x\right)}{\left|a^{3}-a\right||a|^{3 i+3}}\right)
$$

for all $x \in V \backslash\{0\}$.

Proof. First, we define the mappings $J_{m} f: V \rightarrow Y$ by

$$
J_{m} f(x):=\frac{f_{4}\left(a^{m} x\right)}{a^{4 m}}+\frac{f_{3}\left(a^{m} x\right)}{a^{3 m}}+\frac{f_{2}\left(a^{m} x\right)}{a^{2 m}}+\frac{f_{1}\left(a^{m} x\right)}{a^{m}}
$$

for all $x \in V$ and $m \in \mathbb{N}_{0}$. It then follows from (2) and (10) that

$$
\begin{aligned}
& \left\|J_{m} f(x)-J_{m+l} f(x)\right\| \\
& \leq \sum_{i=m}^{m+l-1}\left\|J_{i} f(x)-J_{i+1} f(x)\right\| \\
& =\sum_{i=m}^{m+l-1} \| \frac{f_{e}\left(a^{i+1} x\right)-a^{2} f_{e}\left(a^{i} x\right)}{a^{4 i}\left(a^{4}-a^{2}\right)}-\frac{f_{e}\left(a^{i+2} x\right)-a^{2} f_{e}\left(a^{i+1} x\right)}{a^{4 i+4}\left(a^{4}-a^{2}\right)} \\
& +\frac{f_{o}\left(a^{i+1} x\right)-a f_{o}\left(a^{i} x\right)}{a^{3 i}\left(a^{3}-a\right)}-\frac{f_{o}\left(a^{i+2} x\right)-a f_{o}\left(a^{i+1} x\right)}{a^{3 i+3}\left(a^{3}-a\right)} \\
& \quad+\frac{a^{4} f_{e}\left(a^{i} x\right)-f_{e}\left(a^{i+1} x\right)}{a^{2 i}\left(a^{4}-a^{2}\right)}-\frac{a^{4} f_{e}\left(a^{i+1} x\right)-f_{e}\left(a^{i+2} x\right)}{a^{2 i+2}\left(a^{4}-a^{2}\right)} \\
& \quad+\frac{a^{3} f_{o}\left(a^{i} x\right)-f_{o}\left(a^{i+1} x\right)}{a^{i}\left(a^{3}-a\right)}-\frac{a^{3} f_{o}\left(a^{i+1} x\right)-f_{o}\left(a^{i+2} x\right)}{a^{i+1}\left(a^{3}-a\right)} \| \\
& \sum_{i=m}^{m+l-1} \| \quad \frac{f_{e}\left(a^{i+2} x\right)-\left(a^{2}+a^{4}\right) f_{e}\left(a^{i+1} x\right)+a^{6} f_{e}\left(a^{i} x\right)}{a^{4 i+4}\left(a^{4}-a^{2}\right)} \\
& \quad-\frac{f_{o}\left(a^{i+2} x\right)-\left(a+a^{3}\right) f_{o}\left(a^{i+1} x\right)+a^{4} f_{o}\left(a^{i} x\right)}{a^{3 i+3}\left(a^{3}-a\right)} \\
& \quad+\frac{f_{e}\left(a^{i+2} x\right)-\left(a^{2}+a^{4}\right) f_{e}\left(a^{i+1} x\right)+a^{6} f_{e}\left(a^{i} x\right)}{a^{2 i+2}\left(a^{4}-a^{2}\right)} \\
& \quad+\frac{f_{o}\left(a^{i+2} x\right)-\left(a+a^{3}\right) f_{o}\left(a^{i+1} x\right)+a^{4} f_{o}\left(a^{i} x\right)}{a^{i+1}\left(a^{3}-a\right)} \| \\
& \sum_{i=m}^{m+l-1}\left(\frac{\left|a^{2 i+2}-1\right| \mu\left(a^{i} x\right)}{|a|^{4 i+4}\left|a^{4}-a^{2}\right|}+\frac{\left|a^{2 i+2}-1\right| v\left(a^{i} x\right)}{|a|^{3 i+3}\left|a^{3}-a\right|}\right)
\end{aligned}
$$

for all $x \in V \backslash\{0\}$. 
In view of (8) and (15) and since $|a|>1$, the sequence $\left\{J_{m} f(x)\right\}$ is a Cauchy sequence for all $x \in V \backslash\{0\}$. Since $Y$ is complete and $f(0)=0$, the sequence $\left\{J_{m} f(x)\right\}$ converges for all $x \in V$. Hence, we can define a mapping $F: V \rightarrow Y$ by

$$
F(x):=\lim _{m \rightarrow \infty} J_{m} f(x)=\lim _{m \rightarrow \infty}\left(\frac{f_{4}\left(a^{m} x\right)}{a^{4 m}}+\frac{f_{3}\left(a^{m} x\right)}{a^{3 m}}+\frac{f_{2}\left(a^{m} x\right)}{a^{2 m}}+\frac{f_{1}\left(a^{m} x\right)}{a^{m}}\right)
$$

for all $x \in V$.

If we replace $x$ with $a^{m} x$ in the second inequality of (10) and divide the resulting inequality by $|a|^{m}$, then we obtain

$$
\left\|a^{2} \frac{f_{o}\left(a^{m+2} x\right)}{a^{m+2}}-\left(a^{2}+a^{4}\right) \frac{f_{o}\left(a^{m+1} x\right)}{a^{m+1}}+a^{4} \frac{f_{o}\left(a^{m} x\right)}{a^{m}}\right\| \leq \frac{v\left(a^{m} x\right)}{|a|^{m}}
$$

for all $x \in V \backslash\{0\}$ and $m \in \mathbb{N}$. In view of the second inequalities of (8), this implies that

$$
\lim _{m \rightarrow \infty} \frac{f_{o}\left(a^{m} x\right)}{a^{m}} \text { converges for all } x \in V
$$

because of our hypothesis that $f(0)=0$. Analogously, by the first inequalities of (8) and (10), we obtain

$$
\lim _{m \rightarrow \infty} \frac{f_{e}\left(a^{m} x\right)}{a^{2 m}} \text { converges for all } x \in V .
$$

By (2), (16), (17), and (18), we have

$$
\begin{aligned}
& F_{o}(x)=\lim _{m \rightarrow \infty}\left(\frac{f_{3}\left(a^{m} x\right)}{a^{3 m}}+\frac{f_{1}\left(a^{m} x\right)}{a^{m}}\right)=\lim _{m \rightarrow \infty} \frac{f_{o}\left(a^{m} x\right)}{a^{m}} \\
& F_{e}(x)=\lim _{m \rightarrow \infty}\left(\frac{f_{4}\left(a^{m} x\right)}{a^{4 m}}+\frac{f_{2}\left(a^{m} x\right)}{a^{2 m}}\right)=\lim _{m \rightarrow \infty} \frac{f_{e}\left(a^{m} x\right)}{a^{2 m}}
\end{aligned}
$$

for all $x \in V$.

In view of (2) and (19), we easily obtain

$$
\begin{aligned}
F_{1}(x) & =\frac{a^{3} F_{o}(x)-F_{o}(a x)}{a^{3}-a}=\frac{a^{3}}{a^{3}-a} \lim _{m \rightarrow \infty} \frac{f_{o}\left(a^{m} x\right)}{a^{m}}-\frac{1}{a^{3}-a} \lim _{m \rightarrow \infty} \frac{f_{o}\left(a^{m+1} x\right)}{a^{m}} \\
& =\lim _{m \rightarrow \infty} \frac{f_{o}\left(a^{m} x\right)}{a^{m}}
\end{aligned}
$$

for all $x \in V$. Hence, we obtain

$$
F_{1}(a x)=\lim _{m \rightarrow \infty} \frac{f_{0}\left(a^{m+1} x\right)}{a^{m}}=a F_{1}(x)
$$

for all $x \in V$.

Moreover, by (2) and (19), we obtain

$$
\begin{aligned}
F_{2}(x) & =\frac{a^{4} F_{e}(x)-F_{e}(a x)}{a^{4}-a^{2}}=\frac{a^{4}}{a^{4}-a^{2}} \lim _{m \rightarrow \infty} \frac{f_{e}\left(a^{m} x\right)}{a^{2 m}}-\frac{1}{a^{4}-a^{2}} \lim _{m \rightarrow \infty} \frac{f_{e}\left(a^{m+1} x\right)}{a^{2 m}} \\
& =\lim _{m \rightarrow \infty} \frac{f_{e}\left(a^{m} x\right)}{a^{2 m}}
\end{aligned}
$$

for each $x \in V$. Thus, we obtain

$$
F_{2}(a x)=\lim _{m \rightarrow \infty} \frac{f_{e}\left(a^{m+1} x\right)}{a^{2 m}}=a^{2} F_{2}(x)
$$


for all $x \in V$.

Similarly, using (2), (17), and (19), we have

$$
F_{3}(x)=\frac{F_{o}(a x)-a F_{o}(x)}{a^{3}-a}=\frac{1}{a^{3}-a} \lim _{m \rightarrow \infty} \frac{f_{o}\left(a^{m+1} x\right)}{a^{m}}-\frac{a}{a^{3}-a} \lim _{m \rightarrow \infty} \frac{f_{o}\left(a^{m} x\right)}{a^{m}}=0
$$

for every $x \in V$. Thus, we have $F_{3}(a x)=a^{3} F_{3}(x)$ for all $x \in V$.

Furthermore, by (2), (18), and (19), we obtain

$$
F_{4}(x)=\frac{F_{e}(a x)-a^{2} F_{e}(x)}{a^{4}-a^{2}}=\frac{1}{a^{4}-a^{2}} \lim _{m \rightarrow \infty} \frac{f_{e}\left(a^{m+1} x\right)}{a^{2 m}}-\frac{a^{2}}{a^{4}-a^{2}} \lim _{m \rightarrow \infty} \frac{f_{e}\left(a^{m} x\right)}{a^{2 m}}=0
$$

for all $x \in V$. Therefore, it holds that $F_{4}(a x)=a^{4} F_{4}(x)$ for all $x \in V$.

Now, it follows from (1), (2), and (16) that

$$
\begin{aligned}
& D F\left(x_{1}, x_{2}, \ldots, x_{n}\right) \\
& =\sum_{i=1}^{\ell} c_{i} F\left(a_{i 1} x_{1}+a_{i 2} x_{2}+\cdots+a_{i n} x_{n}\right) \\
& =\lim _{m \rightarrow \infty} \sum_{i=1}^{\ell} c_{i}\left(\frac{f_{4}\left(a^{m}\left(a_{i 1} x_{1}+\cdots+a_{i n} x_{n}\right)\right)}{a^{4 m}}+\frac{f_{3}\left(a^{m}\left(a_{i 1} x_{1}+\cdots+a_{i n} x_{n}\right)\right)}{a^{3 m}}\right. \\
& \left.+\frac{f_{2}\left(a^{m}\left(a_{i 1} x_{1}+\cdots+a_{i n} x_{n}\right)\right)}{a^{2 m}}+\frac{f_{1}\left(a^{m}\left(a_{i 1} x_{1}+\cdots+a_{i n} x_{n}\right)\right)}{a^{m}}\right) \\
& =\lim _{m \rightarrow \infty} \sum_{i=1}^{\ell} c_{i}\left(\frac{f_{e}\left(a^{m+1}\left(a_{i 1} x_{1}+\cdots+a_{i n} x_{n}\right)\right)-a^{2} f_{e}\left(a^{m}\left(a_{i 1} x_{1}+\cdots+a_{i n} x_{n}\right)\right)}{a^{4 m}\left(a^{4}-a^{2}\right)}\right. \\
& +\frac{f_{o}\left(a^{m+1}\left(a_{i 1} x_{1}+\cdots+a_{i n} x_{n}\right)\right)-a f_{o}\left(a^{m}\left(a_{i 1} x_{1}+\cdots+a_{i n} x_{n}\right)\right)}{a^{3 m}\left(a^{3}-a\right)} \\
& +\frac{a^{4} f_{e}\left(a^{m}\left(a_{i 1} x_{1}+\cdots+a_{i n} x_{n}\right)\right)-f_{e}\left(a^{m+1}\left(a_{i 1} x_{1}+\cdots+a_{i n} x_{n}\right)\right)}{a^{2 m}\left(a^{4}-a^{2}\right)} \\
& \left.+\frac{a^{3} f_{o}\left(a^{m}\left(a_{i 1} x_{1}+\cdots+a_{i n} x_{n}\right)\right)-f_{o}\left(a^{m+1}\left(a_{i 1} x_{1}+\cdots+a_{i n} x_{n}\right)\right)}{a^{m}\left(a^{3}-a\right)}\right) \\
& =\lim _{m \rightarrow \infty}\left(\frac{D f_{e}\left(a^{m+1} x_{1}, \ldots, a^{m+1} x_{n}\right)-a^{2} D f_{e}\left(a^{m} x_{1}, \ldots, a^{m} x_{n}\right)}{a^{4 m}\left(a^{4}-a^{2}\right)}\right. \\
& +\frac{D f_{o}\left(a^{m+1} x_{1}, \ldots, a^{m+1} x_{n}\right)-a D f_{o}\left(a^{m} x_{1}, \ldots, a^{m} x_{n}\right)}{a^{3 m}\left(a^{3}-a\right)} \\
& +\frac{a^{4} D f_{e}\left(a^{m} x_{1}, \ldots, a^{m} x_{n}\right)-D f_{e}\left(a^{m+1} x_{1}, \ldots, a^{m+1} x_{n}\right)}{a^{2 m}\left(a^{4}-a^{2}\right)} \\
& \left.+\frac{a^{3} D f_{o}\left(a^{m} x_{1}, \ldots, a^{m} x_{n}\right)-D f_{o}\left(a^{m+1} x_{1}, \ldots, a^{m+1} x_{n}\right)}{a^{m}\left(a^{3}-a\right)}\right)
\end{aligned}
$$

for all $x_{1}, x_{2}, \ldots, x_{n} \in V \backslash\{0\}$.

Hence, in view of (9) and (11), we have 


$$
\begin{aligned}
& \left\|D F\left(x_{1}, x_{2}, \ldots, x_{n}\right)\right\| \\
& \leq \lim _{m \rightarrow \infty}\left(\frac{\left(1+|a|^{m+1}+|a|^{2 m}+|a|^{3 m+1}\right) \varphi_{e}\left(a^{m+1} x_{1}, a^{m+1} x_{2}, \ldots, a^{m+1} x_{n}\right)}{|a|^{4 m}\left|a^{4}-a^{2}\right|}\right. \\
& \left.\quad+\frac{\left(|a|^{2}+|a|^{m+2}+|a|^{2 m+4}+|a|^{3 m+4}\right) \varphi_{e}\left(a^{m} x_{1}, \ldots, a^{m} x_{n}\right)}{|a|^{4 m}\left|a^{4}-a^{2}\right|}\right) \\
& =0
\end{aligned}
$$

for all $x_{1}, x_{2}, \ldots, x_{n} \in V \backslash\{0\}$, where $\varphi_{e}\left(x_{1}, \ldots, x_{n}\right):=\frac{1}{2}\left(\varphi\left(x_{1}, \ldots, x_{n}\right)+\varphi\left(-x_{1}, \ldots,-x_{n}\right)\right)$, i.e., $D F\left(x_{1}, x_{2}, \ldots, x_{n}\right)=0$ for all $x_{1}, x_{2}, \ldots, x_{n} \in V \backslash\{0\}$. Moreover, if we set $m=0$ and let $l \rightarrow \infty$ in (15), then we obtain the inequality (14).

We note that the equalities

$$
\begin{array}{ll}
F_{1}(|a| x)=|a| F_{1}(x), & F_{2}(|a| x)=|a|^{2} F_{2}(x), \\
F_{3}(|a| x)=|a|^{3} F_{3}(x), & F_{4}(|a| x)=|a|^{4} F_{4}(x)
\end{array}
$$

are true in view of (13). (When $a<0$, we have

$$
F_{1}(|a| x)=F_{1}(-a x)=\frac{a^{3} F_{o}(-a x)-F_{o}\left(-a^{2} x\right)}{a^{3}-a}=-\frac{a^{3} F_{o}(a x)-F_{o}\left(a^{2} x\right)}{a^{3}-a},
$$

since $F_{o}$ is odd. By the definition of $F_{1}$ (see (2)) and since we already showed that $F_{1}(a x)=a F_{1}(x)$ for all $x \in V$, we further obtain

$$
F_{1}(|a| x)=-F_{1}(a x)=-a F_{1}(x)=|a| F_{1}(x)
$$

and so on.)

On account of Lemma 1 with $k=|a|$, the mapping $F: V \rightarrow Y$ is the unique mapping satisfying the equalities in (13) and the inequality (14), since the inequality

$$
\begin{aligned}
\|f(x)-F(x)\| & \leq \sum_{i=0}^{\infty}\left(\frac{\left|a^{2 i+2}-1\right| \mu\left(a^{i} x\right)}{\left|a^{4}-a^{2}\right||a|^{4 i+4}}+\frac{\left|a^{2 i+2}-1\right| v\left(a^{i} x\right)}{\left|a^{3}-a\right||a|^{3 i+3}}\right) \\
& \leq \sum_{i=0}^{\infty}\left(\frac{\mu\left(a^{i} x\right)}{\left|a^{2}-1\right||a|^{i}}+\frac{v\left(a^{i} x\right)}{\left|a^{2}-1\right||a|^{i}}\right) \\
& \leq \sum_{i=0}^{\infty} \frac{1}{k^{i}} \phi\left(k^{i} x\right)
\end{aligned}
$$

holds for all $x \in V \backslash\{0\}$, where $k:=|a|$ and $\phi(x):=\frac{1}{\left|a^{2}-1\right|}(\mu(x)+\mu(-x)+v(x)+v(-x))$.

In the following theorem, let $V$ and $Y$ be a real vector space and a real Banach space, respectively.

Theorem 2. Given a real constant a with $|a|>1$, assume that the functions $\mu, v: V \backslash\{0\} \rightarrow[0, \infty)$ satisfy the conditions

$$
\sum_{i=0}^{\infty}|a|^{4 i} \mu\left(\frac{x}{a^{i}}\right)<\infty \text { and } \sum_{i=0}^{\infty}|a|^{4 i} v\left(\frac{x}{a^{i}}\right)<\infty
$$

for all $x \in V \backslash\{0\}$ and a function $\varphi:(V \backslash\{0\})^{n} \rightarrow[0, \infty)$ satisfies the condition

$$
\sum_{i=0}^{\infty}|a|^{4 i} \varphi\left(\frac{x_{1}}{a^{i}}, \frac{x_{2}}{a^{i}}, \ldots, \frac{x_{n}}{a^{i}}\right)<\infty
$$


for all $x_{1}, x_{2}, \ldots, x_{n} \in V \backslash\{0\}$. If a mapping $f: V \rightarrow Y$ satisfies $f(0)=0$ and inequalities in (10) for all $x \in V \backslash\{0\}$, and if $f$ satisfies inequality (11) for all $x_{1}, x_{2}, \ldots, x_{n} \in V \backslash\{0\}$, then there exists a unique mapping $F: V \rightarrow Y$ satisfying (12) for all $x_{1}, x_{2}, \ldots, x_{n} \in V \backslash\{0\}$ and (13) for all $x \in V$, and such that

$$
\|f(x)-F(x)\| \leq \sum_{i=0}^{\infty}\left(\frac{\left|a^{4 i}-a^{2 i}\right|}{\left|a^{4}-a^{2}\right|} \mu\left(\frac{x}{a^{i+1}}\right)+\frac{\left|a^{3 i}-a^{i}\right|}{\left|a^{3}-a\right|} v\left(\frac{x}{a^{i+1}}\right)\right)
$$

for all $x \in V \backslash\{0\}$.

Proof. First, we define the mappings $J_{m} f: V \rightarrow Y$ by

$$
J_{m} f(x):=a^{4 m} f_{4}\left(\frac{x}{a^{m}}\right)+a^{3 m} f_{3}\left(\frac{x}{a^{m}}\right)+a^{2 m} f_{2}\left(\frac{x}{a^{m}}\right)+a^{m} f_{1}\left(\frac{x}{a^{m}}\right)
$$

for all $x \in V$ and $m \in \mathbb{N}_{0}$. It then follows from (2) and (10) that

$$
\begin{aligned}
& \left\|J_{m} f(x)-J_{m+l} f(x)\right\| \\
& \leq \sum_{i=m}^{m+l-1}\left\|J_{i} f(x)-J_{i+1} f(x)\right\| \\
& =\sum_{i=m}^{m+l-1} \| a^{4 i} f_{4}\left(\frac{x}{a^{i}}\right)+a^{3 i} f_{3}\left(\frac{x}{a^{i}}\right)+a^{2 i} f_{2}\left(\frac{x}{a^{i}}\right)+a^{i} f_{1}\left(\frac{x}{a^{i}}\right) \\
& -a^{4 i+4} f_{4}\left(\frac{x}{a^{i+1}}\right)-a^{3 i+3} f_{3}\left(\frac{x}{a^{i+1}}\right)-a^{2 i+2} f_{2}\left(\frac{x}{a^{i+1}}\right)-a^{i+1} f_{1}\left(\frac{x}{a^{i+1}}\right) \| \\
& \leq \frac{1}{\left|a^{4}-a^{2}\right|} \sum_{i=m}^{m+l-1} \| a^{4 i}\left(f_{e}\left(\frac{a^{2} x}{a^{i+1}}\right)-\left(a^{2}+a^{4}\right) f_{e}\left(\frac{a x}{a^{i+1}}\right)+a^{6} f_{e}\left(\frac{x}{a^{i+1}}\right)\right) \\
& -a^{2 i}\left(f_{e}\left(\frac{a^{2} x}{a^{i+1}}\right)-\left(a^{2}+a^{4}\right) f_{e}\left(\frac{a x}{a^{i+1}}\right)+a^{6} f_{e}\left(\frac{x}{a^{i+1}}\right)\right) \| \\
& +\frac{1}{\left|a^{3}-a\right|} \sum_{i=m}^{m+l-1} \| a^{3 i}\left(f_{o}\left(\frac{a^{2} x}{a^{i+1}}\right)-\left(a+a^{3}\right) f_{o}\left(\frac{a x}{a^{i+1}}\right)+a^{4} f_{o}\left(\frac{x}{a^{i+1}}\right)\right) \\
& -a^{i}\left(f_{o}\left(\frac{a^{2} x}{a^{i+1}}\right)-\left(a+a^{3}\right) f_{o}\left(\frac{a x}{a^{i+1}}\right)+a^{4} f_{o}\left(\frac{x}{a^{i+1}}\right)\right) \| \\
& \leq \sum_{i=m}^{m+l-1}\left(\frac{\left|a^{4 i}-a^{2 i}\right|}{\left|a^{4}-a^{2}\right|} \mu\left(\frac{x}{a^{i+1}}\right)+\frac{\left|a^{3 i}-a^{i}\right|}{\left|a^{3}-a\right|} v\left(\frac{x}{a^{i+1}}\right)\right)
\end{aligned}
$$

for all $x \in V \backslash\{0\}$ and $l, m \in \mathbb{N}_{0}$.

On account of (20) and (23), the sequence $\left\{J_{m} f(x)\right\}$ is a Cauchy sequence for all $x \in V \backslash\{0\}$. Since $Y$ is complete and $f(0)=0$, the sequence $\left\{J_{m} f(x)\right\}$ converges for all $x \in V$. Hence, we can define a mapping $F: V \rightarrow Y$ by

$$
\begin{aligned}
F(x) & :=\lim _{m \rightarrow \infty} J_{m} f(x) \\
& =\lim _{m \rightarrow \infty}\left(a^{4 m} f_{4}\left(\frac{x}{a^{m}}\right)+a^{3 m} f_{3}\left(\frac{x}{a^{m}}\right)+a^{2 m} f_{2}\left(\frac{x}{a^{m}}\right)+a^{m} f_{1}\left(\frac{x}{a^{m}}\right)\right)
\end{aligned}
$$

for all $x \in V$. Moreover, if we put $m=0$ and let $l \rightarrow \infty$ in (23), we obtain the inequality (22). 
As we saw in the proof of the previous theorem, it follows from (10) and (20) that

$$
\lim _{m \rightarrow \infty} a^{3 m} f_{o}\left(\frac{x}{a^{m}}\right) \text { and } \lim _{m \rightarrow \infty} a^{4 m} f_{e}\left(\frac{x}{a^{m}}\right) \text { converge for all } x \in V .
$$

By (2) and (25), we obtain

$$
\begin{aligned}
& F_{o}(x)=\lim _{m \rightarrow \infty}\left(a^{3 m} f_{3}\left(\frac{x}{a^{m}}\right)+a^{m} f_{1}\left(\frac{x}{a^{m}}\right)\right)=\lim _{m \rightarrow \infty} a^{3 m} f_{o}\left(\frac{x}{a^{m}}\right), \\
& F_{e}(x)=\lim _{m \rightarrow \infty}\left(a^{4 m} f_{4}\left(\frac{x}{a^{m}}\right)+a^{2 m} f_{2}\left(\frac{x}{a^{m}}\right)\right)=\lim _{m \rightarrow \infty} a^{4 m} f_{e}\left(\frac{x}{a^{m}}\right)
\end{aligned}
$$

for all $x \in V$.

In view of (2), (25), and (26), we have

$$
F_{1}(x)=\frac{a^{3} F_{o}(x)-F_{o}(a x)}{a^{3}-a}=0
$$

for each $x \in V$. Therefore, it holds that $F_{1}(a x)=a F_{1}(x)$ for any $x \in V$. Analogously, it follows from (2), (25), and (26) that $F_{2}(x)=0$ for every $x \in V$. Thus, we have $F_{2}(a x)=a^{2} F_{2}(x)$ for all $x \in V$.

Similarly, using (2), (25), and (26), we obtain

$$
F_{3}(x)=\frac{F_{o}(a x)-a F_{o}(x)}{a^{3}-a}=\lim _{m \rightarrow \infty} a^{3 m} f_{o}\left(\frac{x}{a^{m}}\right)
$$

for all $x \in V$. Hence, we easily see that $F_{3}(a x)=a^{3} F_{3}(x)$ for any $x \in V$. Moreover, similarly as the case of $F_{3}$, we obtain

$$
F_{4}(x)=\frac{F_{e}(a x)-a^{2} F_{e}(x)}{a^{4}-a^{2}}=\lim _{m \rightarrow \infty} a^{4 m} f_{e}\left(\frac{x}{a^{m}}\right)
$$

and $F_{4}(a x)=a^{4} F_{4}(x)$ for any $x \in V$.

Now, using (1), (2), and (24), we have

$$
\begin{aligned}
& D F\left(x_{1}, x_{2}, \ldots, x_{n}\right) \\
& =\sum_{i=1}^{\ell} c_{i} F\left(a_{i 1} x_{1}+a_{i 2} x_{2}+\cdots+a_{i n} x_{n}\right) \\
& =\lim _{m \rightarrow \infty}\left(a^{4 m} \sum_{i=1}^{\ell} c_{i} f_{4}\left(a_{i 1} \frac{x_{1}}{a^{m}}+\cdots+a_{i n} \frac{x_{n}}{a^{m}}\right)\right. \\
& \quad+a^{3 m} \sum_{i=1}^{\ell} c_{i} f_{3}\left(a_{i 1} \frac{x_{1}}{a^{m}}+\cdots+a_{i n} \frac{x_{n}}{a^{m}}\right) \\
& \quad+a^{2 m} \sum_{i=1}^{\ell} c_{i} f_{2}\left(a_{i 1} \frac{x_{1}}{a^{m}}+\cdots+a_{i n} \frac{x_{n}}{a^{m}}\right) \\
& \left.\quad+a^{m} \sum_{i=1}^{\ell} c_{i} f_{1}\left(a_{i 1} \frac{x_{1}}{a^{m}}+\cdots+a_{i n} \frac{x_{n}}{a^{m}}\right)\right)
\end{aligned}
$$




$$
\begin{aligned}
& =\lim _{m \rightarrow \infty}\left[a ^ { 4 m } \sum _ { i = 1 } ^ { \ell } c _ { i } \left(\frac{1}{a^{4}-a^{2}} f_{e}\left(a_{i 1} \frac{x_{1}}{a^{m-1}}+\cdots+a_{i n} \frac{x_{n}}{a^{m-1}}\right)\right.\right. \\
& \left.-\frac{a^{2}}{a^{4}-a^{2}} f_{e}\left(a_{i 1} \frac{x_{1}}{a^{m}}+\cdots+a_{i n} \frac{x_{n}}{a^{m}}\right)\right) \\
& +a^{3 m} \sum_{i=1}^{\ell} c_{i}\left(\frac{1}{a^{3}-a} f_{o}\left(a_{i 1} \frac{x_{1}}{a^{m-1}}+\cdots+a_{i n} \frac{x_{n}}{a^{m-1}}\right)\right. \\
& \left.-\frac{a}{a^{3}-a} f_{o}\left(a_{i 1} \frac{x_{1}}{a^{m}}+\cdots+a_{i n} \frac{x_{n}}{a^{m}}\right)\right) \\
& +a^{2 m} \sum_{i=1}^{\ell} c_{i}\left(\frac{a^{4}}{a^{4}-a^{2}} f_{e}\left(a_{i 1} \frac{x_{1}}{a^{m}}+\cdots+a_{i n} \frac{x_{n}}{a^{m}}\right)\right. \\
& \left.-\frac{1}{a^{4}-a^{2}} f_{e}\left(a_{i 1} \frac{x_{1}}{a^{m-1}}+\cdots+a_{i n} \frac{x_{n}}{a^{m-1}}\right)\right) \\
& +a^{m} \sum_{i=1}^{\ell} c_{i}\left(\frac{a^{3}}{a^{3}-a} f_{o}\left(a_{i 1} \frac{x_{1}}{a^{m}}+\cdots+a_{i n} \frac{x_{n}}{a^{m}}\right)\right. \\
& \left.\left.-\frac{1}{a^{3}-a} f_{o}\left(a_{i 1} \frac{x_{1}}{a^{m-1}}+\cdots+a_{i n} \frac{x_{n}}{a^{m-1}}\right)\right)\right] \\
& =\lim _{m \rightarrow \infty}\left[\frac{a^{4 m}}{a^{4}-a^{2}}\left(D f_{e}\left(\frac{x_{1}}{a^{m-1}}, \ldots, \frac{x_{n}}{a^{m-1}}\right)-a^{2} D f_{e}\left(\frac{x_{1}}{a^{m}}, \ldots, \frac{x_{n}}{a^{m}}\right)\right)\right. \\
& +\frac{a^{3 m}}{a^{3}-a}\left(D f_{o}\left(\frac{x_{1}}{a^{m-1}}, \ldots, \frac{x_{n}}{a^{m-1}}\right)-a D f_{o}\left(\frac{x_{1}}{a^{m}}, \ldots, \frac{x_{n}}{a^{m}}\right)\right) \\
& +\frac{a^{2 m}}{a^{4}-a^{2}}\left(a^{4} D f_{e}\left(\frac{x_{1}}{a^{m}}, \ldots, \frac{x_{n}}{a^{m}}\right)-D f_{e}\left(\frac{x_{1}}{a^{m-1}}, \ldots, \frac{x_{n}}{a^{m-1}}\right)\right) \\
& \left.+\frac{a^{m}}{a^{3}-a}\left(a^{3} D f_{o}\left(\frac{x_{1}}{a^{m}}, \ldots, \frac{x_{n}}{a^{m}}\right)-D f_{o}\left(\frac{x_{1}}{a^{m-1}}, \ldots, \frac{x_{n}}{a^{m-1}}\right)\right)\right]
\end{aligned}
$$

for all $x_{1}, x_{2}, \ldots, x_{n} \in V \backslash\{0\}$. Hence, by (11) and (21), we obtain

$$
\begin{aligned}
& \left\|D F\left(x_{1}, x_{2}, \ldots, x_{n}\right)\right\| \\
& \leq \lim _{m \rightarrow \infty}\left[\left(\frac{|a|^{4 m}+|a|^{2 m}}{\left|a^{4}-a^{2}\right|}+\frac{|a|^{3 m}+|a|^{m}}{\left|a^{3}-a\right|}\right) \varphi_{e}\left(\frac{x_{1}}{a^{m-1}}, \ldots, \frac{x_{n}}{a^{m-1}}\right)\right. \\
& \left.\quad+\left(\frac{|a|^{4 m+2}+|a|^{2 m+4}}{\left|a^{4}-a^{2}\right|}+\frac{|a|^{3 m+1}+|a|^{m+3}}{\left|a^{3}-a\right|}\right) \varphi_{e}\left(\frac{x_{1}}{a^{m}}, \ldots, \frac{x_{n}}{a^{m}}\right)\right] \\
& =0
\end{aligned}
$$

for all $x_{1}, x_{2}, \ldots, x_{n} \in V \backslash\{0\}$, where $\varphi_{e}\left(x_{1}, \ldots, x_{n}\right):=\frac{1}{2}\left(\varphi\left(x_{1}, \ldots, x_{n}\right)+\varphi\left(-x_{1}, \ldots,-x_{n}\right)\right)$. Thus, it holds that $D F\left(x_{1}, x_{2}, \ldots, x_{n}\right)=0$ for all $x_{1}, x_{2}, \ldots, x_{n} \in V \backslash\{0\}$.

As we see in the proof of Theorem 1, we notice that

$$
\begin{array}{ll}
F_{1}(|a| x)=|a| F_{1}(x), & F_{2}(|a| x)=|a|^{2} F_{2}(x), \\
F_{3}(|a| x)=|a|^{3} F_{3}(x), & F_{4}(|a| x)=|a|^{4} F_{4}(x)
\end{array}
$$


for all $x \in V$. According to Lemma 1, the mapping $F: V \rightarrow Y$ satisfying equalities in (13) and inequality (22) is determined uniquely, since

$$
\begin{aligned}
\|f(x)-F(x)\| & \leq \sum_{i=0}^{\infty}\left(\frac{\left|a^{4 i}-a^{2 i}\right|}{\left|a^{4}-a^{2}\right|} \mu\left(\frac{x}{a^{i+1}}\right)+\frac{\left|a^{3 i}-a^{i}\right|}{\left|a^{3}-a\right|} v\left(\frac{x}{a^{i+1}}\right)\right) \\
& \leq \sum_{i=0}^{\infty} \frac{\left|a^{4 i}\right|}{\left|a^{3}-a\right|}\left(\mu\left(\frac{x}{a^{i+1}}\right)+v\left(\frac{x}{a^{i+1}}\right)\right) \\
& \leq \sum_{i=0}^{\infty} k^{4 i} \phi\left(\frac{x}{k^{i}}\right)
\end{aligned}
$$

for all $x \in V \backslash\{0\}$, where $k:=|a|$ and $\phi(x):=\frac{1}{\left|a^{3}-a\right|}\left(\mu\left(\frac{x}{a}\right)+\mu\left(\frac{-x}{a}\right)+v\left(\frac{x}{a}\right)+v\left(\frac{-x}{a}\right)\right)$.

We assume again that $V$ is a real vector space and $Y$ is a real Banach space if there is no specification.

Theorem 3. Given a real constant a with $|a|>1$, assume that the functions $\mu, v: V \backslash\{0\} \rightarrow[0, \infty)$ satisfy all the conditions

$$
\sum_{i=0}^{\infty} \frac{\mu\left(a^{i} x\right)}{|a|^{2 i}}<\infty, \quad \sum_{i=0}^{\infty}|a|^{i} \mu\left(\frac{x}{a^{i}}\right)<\infty, \quad \sum_{i=0}^{\infty} \frac{v\left(a^{i} x\right)}{|a|^{2 i}}<\infty, \quad \sum_{i=0}^{\infty}|a|^{i} v\left(\frac{x}{a^{i}}\right)<\infty
$$

for all $x \in V \backslash\{0\}$ and a function $\varphi:(V \backslash\{0\})^{n} \rightarrow[0, \infty)$ satisfies the conditions

$$
\sum_{i=0}^{\infty} \frac{\varphi\left(a^{i} x_{1}, a^{i} x_{2}, \ldots, a^{i} x_{n}\right)}{|a|^{2 i}}<\infty \text { and } \sum_{i=0}^{\infty}|a|^{i} \varphi\left(\frac{x_{1}}{a^{i}}, \frac{x_{2}}{a^{i}}, \ldots, \frac{x_{n}}{a^{i}}\right)<\infty
$$

for all $x_{1}, x_{2}, \ldots, x_{n} \in V \backslash\{0\}$. If a mapping $f: V \rightarrow Y$ satisfies $f(0)=0$ and inequalities of (10) for all $x \in V \backslash\{0\}$, and if $f$ satisfies inequality (11) for all $x_{1}, x_{2}, \ldots, x_{n} \in V \backslash\{0\}$, then there exists a unique mapping $F: V \rightarrow Y$ satisfying equality (12) for all $x_{1}, x_{2}, \ldots, x_{n} \in V \backslash\{0\}$, equalities in (13) for all $x \in V$, and

$$
\|f(x)-F(x)\| \leq \sum_{i=0}^{\infty} \frac{\mu\left(a^{i} x\right)}{\left|a^{4}-a^{2}\right|} \frac{\left|a^{2 i+2}-1\right|}{|a|^{4 i+4}}+\frac{1}{\left|a^{3}-a\right|} \sum_{i=0}^{\infty}\left(\frac{v\left(a^{i} x\right)}{|a|^{3 i+3}}+|a|^{i} v\left(\frac{x}{a^{i+1}}\right)\right)
$$

for all $x \in V \backslash\{0\}$.

Proof. We define the mappings $J_{m} f: V \rightarrow Y$ by

$$
J_{m} f(x):=\frac{f_{4}\left(a^{m} x\right)}{a^{4 m}}+\frac{f_{3}\left(a^{m} x\right)}{a^{3 m}}+\frac{f_{2}\left(a^{m} x\right)}{a^{2 m}}+a^{m} f_{1}\left(\frac{x}{a^{m}}\right)
$$

for all $x \in V$ and $m \in \mathbb{N}_{0}$. By (2) and (10), we have 


$$
\begin{aligned}
& \left\|J_{m} f(x)-J_{m+l} f(x)\right\| \\
& \leq \sum_{i=m}^{m+l-1}\left\|J_{i} f(x)-J_{i+1} f(x)\right\| \\
& =\sum_{i=m}^{m+l-1} \| \frac{f_{e}\left(a^{i+1} x\right)-a^{2} f_{e}\left(a^{i} x\right)}{a^{4 i}\left(a^{4}-a^{2}\right)}-\frac{f_{e}\left(a^{i+2} x\right)-a^{2} f_{e}\left(a^{i+1} x\right)}{a^{4 i+4}\left(a^{4}-a^{2}\right)} \\
& -\frac{f_{e}\left(a^{i+1} x\right)-a^{4} f_{e}\left(a^{i} x\right)}{a^{2 i}\left(a^{4}-a^{2}\right)}+\frac{f_{e}\left(a^{i+2} x\right)-a^{4} f_{e}\left(a^{i+1} x\right)}{a^{2 i+2}\left(a^{4}-a^{2}\right)} \\
& +\frac{f_{o}\left(a^{i+1} x\right)-a f_{o}\left(a^{i} x\right)}{a^{3 i}\left(a^{3}-a\right)}-\frac{f_{o}\left(a^{i+2} x\right)-a f_{o}\left(a^{i+1} x\right)}{a^{3 i+3}\left(a^{3}-a\right)} \\
& -\frac{a^{i}}{a^{3}-a}\left(f_{o}\left(\frac{a x}{a^{i}}\right)-a^{3} f_{o}\left(\frac{x}{a^{i}}\right)\right) \\
& +\frac{a^{i+1}}{a^{3}-a}\left(f_{o}\left(\frac{a x}{a^{i+1}}\right)-a^{3} f_{o}\left(\frac{x}{a^{i+1}}\right)\right) \| \\
& \leq \frac{1}{\left|a^{4}-a^{2}\right|} \sum_{i=m}^{m+l-1} \|-\frac{f_{e}\left(a^{2} \cdot a^{i} x\right)-\left(a^{2}+a^{4}\right) f_{e}\left(a^{i+1} x\right)+a^{6} f_{e}\left(a^{i} x\right)}{a^{4 i+4}} \\
& +\frac{f_{e}\left(a^{2} \cdot a^{i} x\right)-\left(a^{2}+a^{4}\right) f_{e}\left(a^{i+1} x\right)+a^{6} f_{e}\left(a^{i} x\right)}{a^{2 i+2}} \\
& +\frac{1}{\left|a^{3}-a\right|} \sum_{i=m}^{m+l-1} \|-\frac{f_{o}\left(a^{2} \cdot a^{i} x\right)-\left(a+a^{3}\right) f_{o}\left(a^{i+1} x\right)+a^{4} f_{o}\left(a^{i} x\right)}{a^{3 i+3}} \\
& -a^{i}\left(f_{o}\left(\frac{a^{2} x}{a^{i+1}}\right)-\left(a+a^{3}\right) f_{o}\left(\frac{a x}{a^{i+1}}\right)+a^{4} f_{o}\left(\frac{x}{a^{i+1}}\right)\right) \| \\
& \leq \sum_{i=m}^{m+l-1} \frac{\mu\left(a^{i} x\right)}{\left|a^{4}-a^{2}\right|} \frac{\left|a^{2 i+2}-1\right|}{|a|^{4 i+4}}+\frac{1}{\left|a^{3}-a\right|} \sum_{i=m}^{m+l-1}\left(\frac{v\left(a^{i} x\right)}{|a|^{3 i+3}}+|a|^{i} v\left(\frac{x}{a^{i+1}}\right)\right)
\end{aligned}
$$

for all $x \in V \backslash\{0\}$ and for all $l, m \in \mathbb{N}_{0}$.

In view of (27) and (30), the sequence $\left\{J_{m} f(x)\right\}$ is a Cauchy sequence for all $x \in V \backslash\{0\}$. Since $Y$ is complete and $f(0)=0$, the sequence $\left\{J_{m} f(x)\right\}$ converges for all $x \in V$. Hence, we can define a mapping $F: V \rightarrow Y$ by

$$
F(x):=\lim _{m \rightarrow \infty} J_{m} f(x)=\lim _{m \rightarrow \infty}\left(\frac{f_{4}\left(a^{m} x\right)}{a^{4 m}}+\frac{f_{3}\left(a^{m} x\right)}{a^{3 m}}+\frac{f_{2}\left(a^{m} x\right)}{a^{2 m}}+a^{m} f_{1}\left(\frac{x}{a^{m}}\right)\right)
$$

for all $x \in V$.

By (1), (2), and (31), we obtain 


$$
\begin{aligned}
D F\left(x_{1}, x_{2}, \ldots, x_{n}\right) \\
=\sum_{i=1}^{\ell} c_{i} F\left(a_{i 1} x_{1}+a_{i 2} x_{2}+\cdots+a_{i n} x_{n}\right) \\
=\sum_{i=1}^{\ell} c_{i} \lim _{m \rightarrow \infty}\left(\frac{f_{4}\left(a_{i 1} a^{m} x_{1}+\cdots+a_{i n} a^{m} x_{n}\right)}{a^{4 m}}+\frac{f_{3}\left(a_{i 1} a^{m} x_{1}+\cdots+a_{i n} a^{m} x_{n}\right)}{a^{3 m}}\right. \\
\left.\quad+\frac{f_{2}\left(a_{i 1} a^{m} x_{1}+\cdots+a_{i n} a^{m} x_{n}\right)}{a^{2 m}}+a^{m} f_{1}\left(\frac{a_{i 1} x_{1}}{a^{m}}+\cdots+\frac{a_{i n} x_{n}}{a^{m}}\right)\right) \\
=\lim _{m \rightarrow \infty} \frac{1}{a^{4 m}} \sum_{i=1}^{\ell} c_{i} \frac{f_{e}\left(a_{i 1} a^{m+1} x_{1}+\cdots+a_{i n} a^{m+1} x_{n}\right)-a^{2} f_{e}\left(a_{i 1} a^{m} x_{1}+\cdots+a_{i n} a^{m} x_{n}\right)}{a^{4}-a^{2}} \\
+\lim _{m \rightarrow \infty} \frac{1}{a^{3 m}} \sum_{i=1}^{\ell} c_{i} \frac{f_{o}\left(a_{i 1} a^{m+1} x_{1}+\cdots+a_{i n} a^{m+1} x_{n}\right)-a f_{o}\left(a_{i 1} a^{m} x_{1}+\cdots+a_{i n} a^{m} x_{n}\right)}{a^{3}-a} \\
+\lim _{m \rightarrow \infty} \frac{1}{a^{2 m}} \sum_{i=1}^{\ell} c_{i} \frac{a^{4} f_{e}\left(a_{i 1} a^{m} x_{1}+\cdots+a_{i n} a^{m} x_{n}\right)-f_{e}\left(a_{i 1} a^{m+1} x_{1}+\cdots+a_{i n} a^{m+1} x_{n}\right)}{a^{4}-a^{2}} \\
\quad+\lim _{m \rightarrow \infty} \frac{a^{m}}{a^{3}-a} \sum_{i=1}^{\ell} c_{i}\left(a^{3} f_{o}\left(\frac{a_{i 1} x_{1}}{a^{m}}+\cdots+\frac{a_{i n} x_{n}}{a^{m}}\right)-f_{o}\left(\frac{a_{i 1} x_{1}}{a^{m-1}}+\cdots+\frac{a_{i n} x_{n}}{a^{m-1}}\right)\right) \\
=\lim _{m \rightarrow \infty} \frac{D f_{e}\left(a^{m+1} x_{1}, \ldots, a^{m+1} x_{n}\right)-a^{2} D f_{e}\left(a^{m} x_{1}, \ldots, a^{m} x_{n}\right)}{a^{4 m}\left(a^{4}-a^{2}\right)} \\
\quad+\lim _{m \rightarrow \infty} \frac{D f_{o}\left(a^{m+1} x_{1}, \ldots, a^{m+1} x_{n}\right)-a D f_{o}\left(a^{m} x_{1}, \ldots, a^{m} x_{n}\right)}{a^{3 m}\left(a^{3}-a\right)} \\
+\lim _{m \rightarrow \infty} \frac{a^{4} D f_{e}\left(a^{m} x_{1}, \ldots, a^{m} x_{n}\right)-D f_{e}\left(a^{m+1} x_{1}, \ldots, a^{m+1} x_{n}\right)}{a^{2 m}\left(a^{4}-a^{2}\right)} \\
+\lim _{m \rightarrow \infty} \frac{a^{m}}{a^{3}-a}\left(a^{3} D f_{o}\left(\frac{x_{1}}{a^{m}}, \ldots, \frac{x_{n}}{a^{m}}\right)-D f_{o}\left(\frac{a x_{1}}{a^{m}}, \ldots, \frac{a x_{n}}{a^{m}}\right)\right) \\
=
\end{aligned}
$$

for all $x_{1}, x_{2}, \ldots, x_{n} \in V \backslash\{0\}$. Hence, by (11) and (28), we have

$$
\begin{aligned}
& \left\|D F\left(x_{1}, x_{2}, \ldots, x_{n}\right)\right\| \\
& \leq \lim _{m \rightarrow \infty}\left(\frac{\left(1+|a|^{m+1}+|a|^{2 m}\right) \varphi_{e}\left(a^{m+1} x_{1}, a^{m+1} x_{2}, \ldots, a^{m+1} x_{n}\right)}{|a|^{4 m}\left|a^{4}-a^{2}\right|}\right. \\
& \quad+\frac{\left(a^{2}+|a|^{m+2}+|a|^{2 m+4}\right) \varphi_{e}\left(a^{m} x_{1}, \ldots, a^{m} x_{n}\right)}{|a|^{4 m}\left|a^{4}-a^{2}\right|} \\
& \left.\quad+\frac{|a|^{m+3}}{\left|a^{3}-a\right|} \varphi_{e}\left(\frac{x_{1}}{a^{m}}, \ldots, \frac{x_{n}}{a^{m}}\right)+\frac{|a|^{m}}{\left|a^{3}-a\right|} \varphi_{e}\left(\frac{a x_{1}}{a^{m}}, \ldots, \frac{a x_{n}}{a^{m}}\right)\right) \\
& =0
\end{aligned}
$$

for any $x_{1}, x_{2}, \ldots, x_{n} \in V \backslash\{0\}$, where $\varphi_{e}\left(x_{1}, \ldots, x_{n}\right):=\frac{1}{2}\left(\varphi\left(x_{1}, \ldots, x_{n}\right)+\varphi\left(-x_{1}, \ldots,-x_{n}\right)\right)$, i.e., $D F\left(x_{1}, x_{2}, \ldots, x_{n}\right)=0$ for all $x_{1}, x_{2}, \ldots, x_{n} \in V \backslash\{0\}$. Moreover, if we set $m=0$ and let $l \rightarrow \infty$ in (30), then we obtain the inequality (29).

As we did in the proofs of the previous theorems, on account of (10) and (27), we can show that

$$
\lim _{m \rightarrow \infty} \frac{f_{e}\left(a^{m} x\right)}{a^{2 m}}, \lim _{m \rightarrow \infty} \frac{f_{o}\left(a^{m} x\right)}{a^{3 m}} \text { and } \lim _{m \rightarrow \infty} a^{m} f_{o}\left(\frac{x}{a^{m}}\right) \text { converge for all } x \in V .
$$


In view of (2), (31), and the last relations, we obtain

$$
\begin{aligned}
& F_{e}(x)=\lim _{m \rightarrow \infty} \frac{f_{e}\left(a^{m} x\right)}{a^{2 m}}, \\
& F_{o}(x)=\lim _{m \rightarrow \infty} \frac{f_{o}\left(a^{m} x\right)}{a^{3 m}}+\lim _{m \rightarrow \infty} a^{m} f_{o}\left(\frac{x}{a^{m}}\right)
\end{aligned}
$$

for all $x \in V$.

By using (2) and (32), we obtain

$$
\begin{aligned}
& F_{1}(x)=\frac{a^{3}}{a^{3}-a} F_{o}(x)-\frac{1}{a^{3}-a} F_{o}(a x)=\lim _{m \rightarrow \infty} a^{m} f_{o}\left(\frac{x}{a^{m}}\right), \\
& F_{2}(x)=\frac{a^{4}}{a^{4}-a^{2}} F_{e}(x)-\frac{1}{a^{4}-a^{2}} F_{e}(a x)=\lim _{m \rightarrow \infty} \frac{f_{e}\left(a^{m} x\right)}{a^{2 m}}, \\
& F_{3}(x)=\frac{1}{a^{3}-a} F_{o}(a x)-\frac{a}{a^{3}-a} F_{o}(x)=\lim _{m \rightarrow \infty} \frac{f_{o}\left(a^{m} x\right)}{a^{3 m}}, \\
& F_{4}(x)=\frac{1}{a^{4}-a^{2}} F_{e}(a x)-\frac{a^{2}}{a^{4}-a^{2}} F_{e}(x)=0
\end{aligned}
$$

for all $x \in V$. Hence, we can verify that $F_{1}(a x)=a F_{1}(x), F_{2}(a x)=a^{2} F_{2}(x), F_{3}(a x)=a^{3} F_{3}(x)$, and $F_{4}(a x)=a^{4} F_{4}(x)$ for each $x \in V$.

Similarly as in the proof of Theorem 1, we can use (13) to show that

$$
\begin{array}{ll}
F_{1}(|a| x)=|a| F_{1}(x), & F_{2}(|a| x)=|a|^{2} F_{2}(x), \\
F_{3}(|a| x)=|a|^{3} F_{3}(x), & F_{4}(|a| x)=|a|^{4} F_{4}(x) .
\end{array}
$$

Due to Lemma 2, the mapping $F: V \rightarrow Y$ is the unique mapping satisfying equalities in (13) and inequality (14), since it follows from (29) that

$$
\begin{aligned}
\|f(x)-F(x)\| & \leq \sum_{i=0}^{\infty} \frac{\mu\left(a^{i} x\right)}{\left|a^{4}-a^{2}\right|} \frac{\left|a^{2 i+2}-1\right|}{|a|^{4 i+4}}+\frac{1}{\left|a^{3}-a\right|} \sum_{i=0}^{\infty}\left(\frac{v\left(a^{i} x\right)}{|a|^{3 i+3}}+|a|^{i} v\left(\frac{x}{a^{i+1}}\right)\right) \\
& \leq \sum_{i=0}^{\infty}\left(\frac{\mu\left(a^{i} x\right)+v\left(a^{i} x\right)}{\left|a^{2}-1\right||a|^{2 i}}+\frac{|a|^{i}}{\left|a^{2}-1\right|} v\left(\frac{x}{a^{i+1}}\right)\right) \\
& \leq \sum_{i=0}^{\infty}\left(\frac{1}{k^{2 i}} \psi\left(k^{i} x\right)+k^{i} \phi\left(\frac{x}{k^{i}}\right)\right)
\end{aligned}
$$

for all $x \in V \backslash\{0\}$, where we set $k:=|a|, \psi(x):=\frac{1}{\left|a^{2}-1\right|}(\mu(x)+\mu(-x)+v(x)+v(-x))$ and $\phi(x):=$ $\frac{1}{\left|a^{2}-1\right|}\left(v\left(\frac{x}{a}\right)+v\left(\frac{-x}{a}\right)\right)$.

In the following theorem, let $V$ be a real vector space and $Y$ a real Banach space.

Theorem 4. Assume that the functions $\mu, v: V \backslash\{0\} \rightarrow[0, \infty)$ satisfy the conditions

$$
\begin{aligned}
& \sum_{i=0}^{\infty} \frac{\mu\left(a^{i} x\right)}{|a|^{3 i}}<\infty, \quad \sum_{i=0}^{\infty}|a|^{2 i} \mu\left(\frac{x}{a^{i}}\right)<\infty, \\
& \sum_{i=0}^{\infty} \frac{v\left(a^{i} x\right)}{|a|^{3 i}}<\infty, \quad \sum_{i=0}^{\infty}|a|^{2 i} v\left(\frac{x}{a^{i}}\right)<\infty
\end{aligned}
$$


for all $x \in V \backslash\{0\}$ and a function $\varphi:(V \backslash\{0\})^{n} \rightarrow[0, \infty)$ satisfies the conditions

$$
\sum_{i=0}^{\infty} \frac{\varphi\left(a^{i} x_{1}, a^{i} x_{2}, \ldots, a^{i} x_{n}\right)}{|a|^{3 i}}<\infty \text { and } \sum_{i=0}^{\infty}|a|^{2 i} \varphi\left(\frac{x_{1}}{a^{i}}, \frac{x_{2}}{a^{i}}, \ldots, \frac{x_{n}}{a^{i}}\right)<\infty
$$

for all $x_{1}, x_{2}, \ldots, x_{n} \in V \backslash\{0\}$. If a mapping $f: V \rightarrow Y$ satisfies $f(0)=0$ and the inequality (10) for all $x \in V \backslash\{0\}$ and if $f$ satisfies (11) for all $x_{1}, x_{2}, \ldots, x_{n} \in V \backslash\{0\}$, then there exists a unique mapping $F: V \rightarrow Y$ satisfying the equality (12) for all $x_{1}, x_{2}, \ldots, x_{n} \in V \backslash\{0\}$, the equalities in (13) for all $x \in V$, and

$$
\begin{aligned}
\|f(x)-F(x)\| \leq & \frac{1}{\left|a^{4}-a^{2}\right|} \sum_{i=0}^{\infty}\left(\frac{\mu\left(a^{i} x\right)}{|a|^{4 i+4}}+|a|^{2 i} \mu\left(\frac{x}{a^{i+1}}\right)\right) \\
& +\frac{1}{\left|a^{3}-a\right|} \sum_{i=0}^{\infty}\left(\frac{\nu\left(a^{i} x\right)}{|a|^{3 i+3}}+|a|^{i} v\left(\frac{x}{a^{i+1}}\right)\right)
\end{aligned}
$$

for all $x \in V \backslash\{0\}$.

Proof. We define the mappings $J_{m} f: V \rightarrow Y$ by

$$
J_{m} f(x):=\frac{f_{4}\left(a^{m} x\right)}{a^{4 m}}+\frac{f_{3}\left(a^{m} x\right)}{a^{3 m}}+a^{2 m} f_{2}\left(\frac{x}{a^{m}}\right)+a^{m} f_{1}\left(\frac{x}{a^{m}}\right)
$$

for all $x \in V$ and $m \in \mathbb{N}_{0}$. It then follows from (2) and (10) that

$$
\begin{aligned}
& \left\|J_{m} f(x)-J_{m+l} f(x)\right\| \\
& \leq \sum_{i=m}^{m+l-1}\left\|J_{i} f(x)-J_{i+1} f(x)\right\| \\
& =\sum_{i=m}^{m+l-1} \| \frac{f_{4}\left(a^{i} x\right)}{a^{4 i}}-\frac{f_{4}\left(a^{i+1} x\right)}{a^{4 i+4}}+\frac{f_{3}\left(a^{i} x\right)}{a^{3 i}}-\frac{f_{3}\left(a^{i+1} x\right)}{a^{3 i+3}} \\
& \quad+a^{2 i} f_{2}\left(\frac{x}{a^{i}}\right)-a^{2 i+2} f_{2}\left(\frac{x}{a^{i+1}}\right)+a^{i} f_{1}\left(\frac{x}{a^{i}}\right)-a^{i+1} f_{1}\left(\frac{x}{a^{i+1}}\right) \| \\
& \leq \frac{1}{\left|a^{4}-a^{2}\right|} \sum_{i=m}^{m+l-1} \|-\frac{f_{e}\left(a^{2} \cdot a^{i} x\right)-\left(a^{2}+a^{4}\right) f_{e}\left(a^{i+1} x\right)+a^{6} f_{e}\left(a^{i} x\right)}{a^{4 i+4}} \\
& +\frac{1}{\left|a^{3}-a\right|} \sum_{i=m}^{m+l-1} \|-\frac{f_{o}\left(a^{2} \cdot a^{i} x\right)-\left(a+a^{3}\right) f_{o}\left(a^{i+1} x\right)+a^{4} f_{o}\left(a^{i} x\right)}{a^{3 i+3}} \\
& \left.\leq \frac{1}{\left|a^{4}-a^{2}\right|} \sum_{i=m}^{m+l-1}\left(\frac{a^{2} x}{a^{i+1}}\right)-\left(a^{2}+a^{4}\right) f_{e}\left(\frac{a x}{a^{i+1}}\right)+a^{6} f_{e}\left(\frac{x}{a^{i+1}}\right)\right) \| \\
& +\frac{1}{\left.\left|a^{3}-a\right| a^{i+1} x\right)} \sum_{i=m}^{m+l-1}\left(\frac{v\left(a^{i} x\right)}{|a|^{3 i+3}}+|a|^{i} v\left(\frac{x}{a^{i+1}}\right)\right)
\end{aligned}
$$

for all $x \in V \backslash\{0\}$. 
In view of (33) and (36), the sequence $\left\{J_{m} f(x)\right\}$ is a Cauchy sequence for all $x \in V \backslash\{0\}$. Since $Y$ is complete and $f(0)=0$, the sequence $\left\{J_{m} f(x)\right\}$ converges for all $x \in V$. Hence, we can define a mapping $F: V \rightarrow Y$ by

$$
F(x):=\lim _{m \rightarrow \infty} J_{m} f(x)=\lim _{m \rightarrow \infty}\left(\frac{f_{4}\left(a^{m} x\right)}{a^{4 m}}+\frac{f_{3}\left(a^{m} x\right)}{a^{3 m}}+a^{2 m} f_{2}\left(\frac{x}{a^{m}}\right)+a^{m} f_{1}\left(\frac{x}{a^{m}}\right)\right)
$$

for all $x \in V$. Then, by oddness and evenness of $F_{o}$ and $F_{e}$, it follows from (37) that

$$
\begin{aligned}
& F_{o}(x)=\lim _{m \rightarrow \infty}\left(\frac{f_{3}\left(a^{m} x\right)}{a^{3 m}}+a^{m} f_{1}\left(\frac{x}{a^{m}}\right)\right), \\
& F_{e}(x)=\lim _{m \rightarrow \infty}\left(\frac{f_{4}\left(a^{m} x\right)}{a^{4 m}}+a^{2 m} f_{2}\left(\frac{x}{a^{m}}\right)\right)
\end{aligned}
$$

for all $x \in V$. Moreover, if we set $m=0$ and let $l \rightarrow \infty$ in (36), we obtain the inequality (35).

In view of (10) and (33), it holds that

$$
\lim _{m \rightarrow \infty} a^{m} f_{o}\left(\frac{x}{a^{m}}\right), \lim _{m \rightarrow \infty} \frac{f_{o}\left(a^{m} x\right)}{a^{3 m}}, \lim _{m \rightarrow \infty} a^{2 m} f_{e}\left(\frac{x}{a^{m}}\right), \lim _{m \rightarrow \infty} \frac{f_{e}\left(a^{m} x\right)}{a^{4 m}}
$$

converge for all $x \in V$. Using these facts, we obtain

$$
\begin{aligned}
F_{1}(x)= & \frac{a^{3}}{a^{3}-a} F_{o}(x)-\frac{1}{a^{3}-a} F_{o}(a x) \\
= & \frac{a^{3}}{a^{3}-a} \lim _{m \rightarrow \infty}\left(\frac{f_{3}\left(a^{m} x\right)}{a^{3 m}}+a^{m} f_{1}\left(\frac{x}{a^{m}}\right)\right) \\
& -\frac{1}{a^{3}-a} \lim _{m \rightarrow \infty}\left(\frac{f_{3}\left(a^{m+1} x\right)}{a^{3 m}}+a^{m} f_{1}\left(\frac{x}{a^{m-1}}\right)\right) \\
= & \frac{a^{3}}{a^{3}-a} \lim _{m \rightarrow \infty} a^{m} f_{1}\left(\frac{x}{a^{m}}\right)-\frac{a}{a^{3}-a} \lim _{m \rightarrow \infty} a^{m-1} f_{1}\left(\frac{x}{a^{m-1}}\right) \\
= & \lim _{m \rightarrow \infty} a^{m} f_{1}\left(\frac{x}{a^{m}}\right)
\end{aligned}
$$

for all $x \in V$. Therefore, $F_{1}(a x)=a F_{1}(x)$ for all $x \in V$. By the similar way, we can show that the equalities in (13) hold true for any $x \in V$.

On account of (1), (2), and (37), it holds that

$$
\begin{aligned}
& D F\left(x_{1}, x_{2}, \ldots, x_{n}\right) \\
& =\sum_{i=1}^{\ell} c_{i} F\left(a_{i 1} x_{1}+a_{i 2} x_{2}+\cdots+a_{i n} x_{n}\right) \\
& =\sum_{i=1}^{\ell} c_{i} \lim _{m \rightarrow \infty}\left(\frac{1}{a^{4 m}} f_{4}\left(a^{m}\left(a_{i 1} x_{1}+\cdots+a_{i n} x_{n}\right)\right)\right. \\
& +\frac{1}{a^{3 m}} f_{3}\left(a^{m}\left(a_{i 1} x_{1}+\cdots+a_{i n} x_{n}\right)\right) \\
& +a^{2 m} f_{2}\left(\frac{a_{i 1} x_{1}+\cdots+a_{i n} x_{n}}{a^{m}}\right)
\end{aligned}
$$




$$
\left.\left.\begin{array}{r}
\left.+a^{m} f_{1}\left(\frac{a_{i 1} x_{1}+\ldots+a_{i n} x_{n}}{a^{m}}\right)\right) \\
=\lim _{m \rightarrow \infty} \frac{1}{a^{4 m}}\left(\frac{1}{a^{4}-a^{2}} D f_{e}\left(a^{m+1} x_{1}, \ldots, a^{m+1} x_{n}\right)\right. \\
\left.+\frac{a^{2}}{a^{4}-a^{2}} D f_{e}\left(a^{m} x_{1}, \ldots, a^{m} x_{n}\right)\right) \\
+\lim _{m \rightarrow \infty} \frac{1}{a^{3 m}}\left(\frac{1}{a^{3}-a} D f_{o}\left(a^{m+1} x_{1}, \ldots, a^{m+1} x_{n}\right)\right. \\
+\lim _{m \rightarrow \infty} a^{2 m}\left(\frac{a}{a^{3}-a} D f_{o}\left(a^{m} x_{1}, \ldots, a^{m} x_{n}\right)\right) \\
\frac{a^{4}-a^{2}}{a^{m}}\left(\frac{x_{e}}{a^{m}}, \ldots, \frac{x_{n}}{a^{m}}\right) \\
\frac{1}{a^{3}-a} D f_{o}\left(\frac{x_{1}}{a^{m}}, \ldots, \frac{x_{n}}{a^{m}}\right) \\
-\frac{1}{a^{3}-a} D f_{o}\left(\frac{x_{1}}{a^{m-1}}, \ldots, \frac{x_{n}}{a^{m-1}}\right)
\end{array}\right)\right)
$$

for all $x_{1}, x_{2}, \ldots, x_{n} \in V \backslash\{0\}$.

Using (11) and (34), we obtain

$$
\begin{aligned}
\left\|D F\left(x_{1}, x_{2}, \ldots, x_{n}\right)\right\| \\
\leq \lim _{m \rightarrow \infty}\left(\frac{1}{\left|a^{4}-a^{2}\right||a|^{4 m}} \varphi_{e}\left(a^{m+1} x_{1}, \ldots, a^{m+1} x_{n}\right)\right. \\
+\frac{|a|^{2}}{\left|a^{4}-a^{2}\right||a|^{4 m}} \varphi_{e}\left(a^{m} x_{1}, \ldots, a^{m} x_{n}\right) \\
+\frac{1}{\left|a^{3}-a\right||a|^{3 m}} \varphi_{e}\left(a^{m+1} x_{1}, \ldots, a^{m+1} x_{n}\right) \\
+\frac{|a|}{\left|a^{3}-a\right||a|^{3 m}} \varphi_{e}\left(a^{m} x_{1}, \ldots, a^{m} x_{n}\right) \\
+\frac{|a|^{4}|a|^{2 m}}{\left|a^{4}-a^{2}\right|} \varphi_{e}\left(\frac{x_{1}}{a^{m}}, \ldots, \frac{x_{n}}{a^{m}}\right)+\frac{|a|^{2 m}}{\left|a^{4}-a^{2}\right|} \varphi_{e}\left(\frac{x_{1}}{a^{m-1}}, \ldots, \frac{x_{n}}{a^{m-1}}\right) \\
\left.+\frac{|a|^{3}|a|^{m}}{\left|a^{3}-a\right|} \varphi_{e}\left(\frac{x_{1}}{a^{m}}, \ldots, \frac{x_{n}}{a^{m}}\right)+\frac{|a|^{m}}{\left|a^{3}-a\right|} \varphi_{e}\left(\frac{x_{1}}{a^{m-1}}, \ldots, \frac{x_{n}}{a^{m-1}}\right)\right) \\
=0 \quad
\end{aligned}
$$

for all $x_{1}, x_{2}, \ldots, x_{n} \in V \backslash\{0\}$, i.e., $D F\left(x_{1}, x_{2}, \ldots, x_{n}\right)=0$ for all $x_{1}, x_{2}, \ldots, x_{n} \in V \backslash\{0\}$. By a similar way as the previous proofs, we notice that the equalities

$$
\begin{array}{ll}
F_{1}(|a| x)=|a| F_{1}(x), & F_{2}(|a| x)=|a|^{2} F_{2}(x), \\
F_{3}(|a| x)=|a|^{3} F_{3}(x), & F_{4}(|a| x)=|a|^{4} F_{4}(x)
\end{array}
$$

are true in view of (13). 
Using Lemma 3, we conclude that the mapping $F: V \rightarrow Y$ is the unique mapping satisfying equalities in (13) and the inequality (35), since

$$
\begin{aligned}
& \|f(x)-F(x)\| \\
& \leq \frac{1}{\left|a^{4}-a^{2}\right|} \sum_{i=0}^{\infty}\left(\frac{\mu\left(a^{i} x\right)}{|a|^{4 i+4}}+|a|^{2 i} \mu\left(\frac{x}{a^{i+1}}\right)\right)+\frac{1}{\left|a^{3}-a\right|} \sum_{i=0}^{\infty}\left(\frac{v\left(a^{i} x\right)}{|a|^{3 i+3}}+|a|^{i} v\left(\frac{x}{a^{i+1}}\right)\right) \\
& \leq \frac{1}{\left|a^{2}-1\right|} \sum_{i=0}^{\infty}\left(\frac{\mu\left(a^{i} x\right)+v\left(a^{i} x\right)}{|a|^{3 i}}+|a|^{2 i} \mu\left(\frac{x}{a^{i+1}}\right)+|a|^{2 i} v\left(\frac{x}{a^{i+1}}\right)\right) \\
& \leq \sum_{i=0}^{\infty}\left(\frac{1}{k^{3 i}} \psi\left(k^{i} x\right)+k^{2 i} \phi\left(\frac{x}{k^{i}}\right)\right)
\end{aligned}
$$

for all $x \in V \backslash\{0\}$, where we set $k:=|a|, \psi(x):=\frac{1}{\left|a^{2}-1\right|}(\mu(x)+\mu(-x)+v(x)+v(-x))$ and $\phi(x):=$ $\frac{1}{\left|a^{2}-1\right|}\left(\mu\left(\frac{x}{a}\right)+\mu\left(\frac{-x}{a}\right)+v\left(\frac{x}{a}\right)+v\left(\frac{-x}{a}\right)\right)$.

Theorem 5. Assume that the functions $\mu, v: V \backslash\{0\} \rightarrow[0, \infty)$ satisfy all the conditions

$$
\sum_{i=0}^{\infty}|a|^{3 i} \mu\left(\frac{x}{a^{i}}\right)<\infty, \quad \sum_{i=0}^{\infty} \frac{\mu\left(a^{i} x\right)}{|a|^{4 i}}<\infty, \quad \sum_{i=0}^{\infty}|a|^{3 i} v\left(\frac{x}{a^{i}}\right)<\infty, \quad \sum_{i=0}^{\infty} \frac{v\left(a^{i} x\right)}{|a|^{4 i}}<\infty
$$

for all $x \in V \backslash\{0\}$ and a function $\varphi:(V \backslash\{0\})^{n} \rightarrow[0, \infty)$ satisfies the conditions

$$
\sum_{i=0}^{\infty} \frac{\varphi\left(a^{i} x_{1}, a^{i} x_{2}, \ldots, a^{i} x_{n}\right)}{|a|^{4 i}}<\infty \text { and } \sum_{i=0}^{\infty}|a|^{3 i} \varphi\left(\frac{x_{1}}{a^{i}}, \frac{x_{2}}{a^{i}}, \ldots, \frac{x_{n}}{a^{i}}\right)<\infty
$$

for all $x_{1}, x_{2}, \ldots, x_{n} \in V \backslash\{0\}$. If a mapping $f: V \rightarrow Y$ satisfies $f(0)=0$ and the inequality (10) for all $x \in V \backslash\{0\}$, and if $f$ satisfies (11) for all $x_{1}, x_{2}, \ldots, x_{n} \in V \backslash\{0\}$, then there exists a unique mapping $F: V \rightarrow Y$ satisfying the equality (12) for all $x_{1}, x_{2}, \ldots, x_{n} \in V \backslash\{0\}$, the equalities in (13) for all $x \in V$, and

$$
\|f(x)-F(x)\| \leq \frac{1}{\left|a^{4}-a^{2}\right|} \sum_{i=0}^{\infty}\left(\frac{\mu\left(a^{i} x\right)}{|a|^{4(i+1)}}+|a|^{2 i} \mu\left(\frac{x}{a^{i+1}}\right)\right)+\sum_{i=0}^{\infty} \frac{\left|a^{3 i}-a^{i}\right|}{\left|a^{3}-a\right|} v\left(\frac{x}{a^{i+1}}\right)
$$

for all $x \in V \backslash\{0\}$.

Proof. We define the mappings $J_{m} f: V \rightarrow Y$ by

$$
J_{m} f(x):=\frac{f_{4}\left(a^{m} x\right)}{a^{4 m}}+a^{3 m} f_{3}\left(\frac{x}{a^{m}}\right)+a^{2 m} f_{2}\left(\frac{x}{a^{m}}\right)+a^{m} f_{1}\left(\frac{x}{a^{m}}\right)
$$

for all $x \in V$ and $m \in \mathbb{N}_{0}$. It follows from (2) and (10) that 


$$
\begin{aligned}
& \left\|J_{m} f(x)-J_{m+l} f(x)\right\| \\
& \leq \sum_{i=m}^{m+l-1}\left\|J_{i} f(x)-J_{i+1} f(x)\right\| \\
& =\sum_{i=m}^{m+l-1} \| \frac{f_{4}\left(a^{i} x\right)}{a^{4 i}}-\frac{f_{4}\left(a^{i+1} x\right)}{a^{4 i+4}}+a^{2 i} f_{2}\left(\frac{x}{a^{i}}\right)-a^{2 i+2} f_{2}\left(\frac{x}{a^{i+1}}\right) \\
& +a^{3 i} f_{3}\left(\frac{x}{a^{i}}\right)-a^{3 i+3} f_{3}\left(\frac{x}{a^{i+1}}\right)+a^{i} f_{1}\left(\frac{x}{a^{i}}\right)-a^{i+1} f_{1}\left(\frac{x}{a^{i+1}}\right) \| \\
& \leq \frac{1}{\left|a^{4}-a^{2}\right|} \sum_{i=m}^{m+l-1} \|-\frac{f_{e}\left(a^{2} \cdot a^{i} x\right)-\left(a^{2}+a^{4}\right) f_{e}\left(a^{i+1} x\right)+a^{6} f_{e}\left(a^{i} x\right)}{a^{4 i+4}} \\
& +\frac{1}{\left|a^{3}-a\right|} \sum_{i=m}^{m+l-1} \| a^{3 i}\left(f_{o}\left(\frac{a^{2} x}{a^{i+1}}\right)-\left(a+a^{3}\right) f_{o}\left(\frac{a x}{a^{i+1}}\right)+a^{4} f_{o}\left(\frac{x}{a^{i+1}}\right)\right) \\
& \left.\leq \frac{1}{\left|a^{4}-a^{2}\right|} \sum_{i=m}^{m+l-1}\left(\frac{\mu\left(a^{2} x\right)}{|a|^{4(i+1)}}+|a|^{4}\right) f_{e}\left(\frac{a x}{a^{i+1}}\right)+a^{6} f_{e}\left(\frac{x}{a^{i+1}}\right)\right) \| \\
& \left.\left.a^{i+1}\right)\right)+\sum_{i=m}^{m+l-1} \frac{\left|a^{3 i}-a^{i}\right|}{\left|a^{3}-a\right|} v\left(\frac{x}{a^{i+1}}\right)
\end{aligned}
$$

for all $x \in V \backslash\{0\}$ and $l, m \in \mathbb{N}_{0}$.

On the other hand, by (10) and (38), we further see that

$$
\lim _{m \rightarrow \infty} a^{3 m} f_{o}\left(\frac{x}{a^{m}}\right), \quad \lim _{m \rightarrow \infty} a^{3 m} f_{e}\left(\frac{x}{a^{m}}\right), \quad \lim _{m \rightarrow \infty} \frac{f_{e}\left(a^{m} x\right)}{a^{4 m}} \text { converge for all } x \in V .
$$

In view of (38) and (41), the sequence $\left\{J_{m} f(x)\right\}$ is a Cauchy sequence for all $x \in V \backslash\{0\}$. Since $Y$ is complete and $f(0)=0$, the sequence $\left\{J_{m} f(x)\right\}$ converges for all $x \in V$. Hence, considering (2) and (42), we can define a mapping $F: V \rightarrow Y$ by

$$
F(x):=\lim _{m \rightarrow \infty} J_{m} f(x)=\lim _{m \rightarrow \infty}\left(\frac{f_{4}\left(a^{m} x\right)}{a^{4 m}}+a^{3 m} f_{3}\left(\frac{x}{a^{m}}\right)\right)
$$

for all $x \in V$. Moreover, if we set $m=0$ and let $l \rightarrow \infty$ in (41), we obtain inequality (40).

On account of (2) and (43), the oddness and evenness of $F_{o}$ and $F_{e}$ imply that

$$
F_{0}(x)=\lim _{m \rightarrow \infty} a^{3 m} f_{3}\left(\frac{x}{a^{m}}\right) \text { and } F_{e}(x)=\lim _{m \rightarrow \infty} \frac{f_{4}\left(a^{m} x\right)}{a^{4 m}}
$$

for all $x \in V$. Using these facts, for example, we obtain

$$
\begin{aligned}
F_{4}(x) & =\frac{1}{a^{4}-a^{2}} F_{e}(a x)-\frac{a^{2}}{a^{4}-a^{2}} F_{e}(x) \\
& =\frac{1}{a^{4}-a^{2}} \lim _{m \rightarrow \infty} \frac{f_{4}\left(a^{m+1} x\right)}{a^{4 m}}-\frac{a^{2}}{a^{4}-a^{2}} \lim _{m \rightarrow \infty} \frac{f_{4}\left(a^{m} x\right)}{a^{4 m}} \\
& =\lim _{m \rightarrow \infty} \frac{f_{4}\left(a^{m} x\right)}{a^{4 m}}
\end{aligned}
$$

for all $x \in V$. Therefore, $F_{4}(a x)=a^{4} F_{4}(x)$ for all $x \in V$. Similarly, we can show that all the equalities in (13) hold true for any $x \in V$. 
By using (1), (2), and (43), we have

$$
\begin{aligned}
& D F\left(x_{1}, x_{2}, \ldots, x_{n}\right) \\
& =\sum_{i=1}^{\ell} c_{i} F\left(a_{i 1} x_{1}+a_{i 2} x_{2}+\cdots+a_{i n} x_{n}\right) \\
& =\sum_{i=1}^{\ell} c_{i} \lim _{m \rightarrow \infty}\left(\frac{1}{a^{4 m}} f_{4}\left(a^{m}\left(a_{i 1} x_{1}+\cdots+a_{i n} x_{n}\right)\right)\right. \\
& \left.+a^{3 m} f_{3}\left(\frac{a_{i 1} x_{1}+\cdots+a_{i n} x_{n}}{a^{m}}\right)\right) \\
& =\sum_{i=1}^{\ell} c_{i} \lim _{m \rightarrow \infty}\left(\frac{1}{\left(a^{4}-a^{2}\right) a^{4 m}} f_{e}\left(a^{m+1}\left(a_{i 1} x_{1}+\cdots+a_{i n} x_{n}\right)\right)\right. \\
& \left.-\frac{a^{2}}{\left(a^{4}-a^{2}\right) a^{4 m}} f_{e}\left(a^{m}\left(a_{i 1} x_{1}+\cdots+a_{i n} x_{n}\right)\right)\right) \\
& +\sum_{i=1}^{\ell} c_{i} \lim _{m \rightarrow \infty}\left(\frac{a^{3 m}}{a^{3}-a} f_{o}\left(\frac{a_{i 1} x_{1}+\cdots+a_{i n} x_{n}}{a^{m-1}}\right)\right. \\
& \left.-\frac{a^{3 m+1}}{a^{3}-a} f_{o}\left(\frac{a_{i 1} x_{1}+\cdots+a_{i n} x_{n}}{a^{m}}\right)\right) \\
& =\lim _{m \rightarrow \infty}\left(\frac{1}{\left(a^{4}-a^{2}\right) a^{4 m}} D f_{e}\left(a^{m+1} x_{1}, \ldots, a^{m+1} x_{n}\right)\right. \\
& \left.-\frac{a^{2}}{\left(a^{4}-a^{2}\right) a^{4 m}} D f_{e}\left(a^{m} x_{1}, \ldots, a^{m} x_{n}\right)\right) \\
& +\lim _{m \rightarrow \infty}\left(\frac{a^{3 m}}{a^{3}-a} D f_{o}\left(\frac{x_{1}}{a^{m-1}}, \ldots, \frac{x_{n}}{a^{m-1}}\right)-\frac{a^{3 m+1}}{a^{3}-a} D f_{o}\left(\frac{x_{1}}{a^{m}}, \ldots, \frac{x_{n}}{a^{m}}\right)\right)
\end{aligned}
$$

for all $x_{1}, x_{2}, \ldots, x_{n} \in V \backslash\{0\}$.

By (11) and (39), together with the last equality, we obtain

$$
\left\|D F\left(x_{1}, x_{2}, \ldots, x_{n}\right)\right\|=0
$$

for all $x_{1}, x_{2}, \ldots, x_{n} \in V \backslash\{0\}$, i.e., $D F\left(x_{1}, x_{2}, \ldots, x_{n}\right)=0$ for all $x_{1}, x_{2}, \ldots, x_{n} \in V \backslash\{0\}$.

We remark that the equalities

$$
\begin{array}{ll}
F_{1}(|a| x)=|a| F_{1}(x), & F_{2}(|a| x)=|a|^{2} F_{2}(x), \\
F_{3}(|a| x)=|a|^{3} F_{3}(x), & F_{4}(|a| x)=|a|^{4} F_{4}(x)
\end{array}
$$

are true in view of (13).

Using Lemma 4, we conclude that the mapping $F: V \rightarrow Y$ is the unique mapping satisfying equalities in (13) and the inequality (40), since

$$
\begin{aligned}
\|f(x)-F(x)\| & \leq \frac{1}{\left|a^{4}-a^{2}\right|} \sum_{i=0}^{\infty}\left(\frac{\mu\left(a^{i} x\right)}{|a|^{4(i+1)}}+|a|^{2 i} \mu\left(\frac{x}{a^{i+1}}\right)\right)+\sum_{i=0}^{\infty} \frac{\left|a^{3 i}-a^{i}\right|}{\left|a^{3}-a\right|} \nu\left(\frac{x}{a^{i+1}}\right) \\
& \leq \sum_{i=0}^{\infty}\left(\frac{\mu\left(a^{i} x\right)}{|a|^{4 i}\left|a^{2}-1\right|}+\frac{|a|^{3 i}}{\left|a^{2}-1\right|} \mu\left(\frac{x}{a^{i+1}}\right)+\frac{|a|^{3 i}}{\left|a^{2}-1\right|} v\left(\frac{x}{a^{i+1}}\right)\right) \\
& \leq \sum_{i=0}^{\infty}\left(\frac{1}{k^{4 i}} \psi\left(k^{i} x\right)+k^{3 i} \phi\left(\frac{x}{k^{i}}\right)\right)
\end{aligned}
$$


for all $x \in V \backslash\{0\}$, where we set $k:=|a|, \phi(x):=\frac{1}{\left|a^{2}-1\right|}\left(\mu\left(\frac{x}{a}\right)+\mu\left(\frac{-x}{a}\right)+v\left(\frac{x}{a}\right)+v\left(\frac{-x}{a}\right)\right)$ and $\psi(x):=$ $\frac{1}{\left|a^{2}-1\right|}(\mu(x)+\mu(-x))$.

\section{Corollaries}

By using Theorems 1-5, we can prove the Hyers-Ulam-Rassias stability of the functional Equation (1).

Corollary 1. Assume that $X$ is a real normed space, $Y$ is a real Banach space, and $p, \theta, \eta$, and $\xi$ are real constants such that $p \notin\{1,2,3,4\},|a|>1, \xi>0, \eta>0$, and $\theta>0$. If a mapping $f: X \rightarrow Y$ satisfies $f(0)=0$ and

$$
\begin{aligned}
\left\|f_{e}\left(a^{2} x\right)-\left(a^{2}+a^{4}\right) f_{e}(a x)+a^{6} f_{e}(x)\right\| & \leq \eta\|x\|^{p}, \\
\left\|f_{o}\left(a^{2} x\right)-\left(a+a^{3}\right) f_{o}(a x)+a^{4} f_{o}(x)\right\| & \leq \xi\|x\|^{p}
\end{aligned}
$$

for all $x \in X \backslash\{0\}$ and if $f$ moreover satisfies the inequality

$$
\left\|D f\left(x_{1}, x_{2}, \ldots, x_{n}\right)\right\| \leq \theta\left(\left\|x_{1}\right\|^{p}+\cdots+\left\|x_{n}\right\|^{p}\right)
$$

for all $x_{1}, x_{2}, \ldots, x_{n} \in X \backslash\{0\}$, then there exists a unique mapping $F: X \rightarrow Y$ satisfying (12) for all $x_{1}, x_{2}, \ldots, x_{n} \in X \backslash\{0\}$, and the equalities in (13) for all $x \in X$, as well as

$$
\|f(x)-F(x)\| \leq \frac{\eta\|x\|^{p}}{\left.\left|a^{4}-\right| a\right|^{p}|||a|^{p}-a^{2} \mid}+\frac{\xi\|x\|^{p}}{\|\left. a\right|^{3}-|a|^{p}|||a|^{p}-|a| \mid}
$$

for all $x \in X \backslash\{0\}$.

Proof. If we set $\varphi\left(x_{1}, x_{2}, \ldots, x_{n}\right):=\theta\left(\left\|x_{1}\right\|^{p}+\cdots+\left\|x_{n}\right\|^{p}\right)$ for all $x_{1}, x_{2}, \ldots, x_{n} \in X \backslash\{0\}$, then $\varphi$ satisfies (9), (21), (28), (34), and (44) when $p<1, p>4,1<p<2,2<p<3$, and $3<p<4$, respectively.

Therefore, there is a unique mapping $F: X \rightarrow Y$ satisfying (12) for all $x_{1}, x_{2}, \ldots, x_{n} \in X \backslash\{0\}$, the equalities in (13) for all $x \in X$, as well as

$$
\begin{aligned}
& \|f(x)-F(x)\| \\
& \quad \leq \begin{cases}\left(\frac{\eta}{|a|^{2}-|a|^{p}}-\frac{\eta}{|a|^{4}-|a|^{p}}+\frac{|a| \xi}{|a|-|a|^{p}}-\frac{|a| \xi}{|a|^{3}-|a|^{p}}\right) \frac{\|x\|^{p}}{|a|^{2}\left|a^{2}-1\right|} \quad(\text { for } p<1), \\
\left(\frac{\eta}{|a|^{2}-|a|^{p}}-\frac{\eta}{|a|^{4}-|a|^{p}}+\frac{|a| \xi}{|a|^{p}-|a|}+\frac{|a| \xi}{|a|^{3}-|a|^{p}}\right) \frac{\|x\|^{p}}{|a|^{2}\left|a^{2}-1\right|} \quad(\text { for } 1<p<2), \\
\left(\frac{\eta}{|a|^{p}-|a|^{2}}+\frac{\eta}{|a|^{4}-|a|^{p}}+\frac{|a| \xi}{|a|^{p}-|a|}+\frac{|a| \xi}{|a|^{3}-|a|^{p}}\right) \frac{\|x\|^{p}}{|a|^{2}\left|a^{2}-1\right|} \quad(\text { for } 2<p<3), \\
\left(\frac{\eta}{|a|^{p}-|a|^{2}}+\frac{\eta}{|a|^{4}-|a|^{p}}+\frac{|a| \xi}{|a|^{p}-|a|^{3}}-\frac{|a| \xi}{|a|^{p}-|a|}\right) \frac{\|x\|^{p}}{|a|^{2}\left|a^{2}-1\right|} \quad(\text { for } 3<p<4), \\
\left(\frac{\eta}{|a|^{p}-|a|^{4}}-\frac{\eta}{|a|^{p}-|a|^{2}}+\frac{|a| \xi}{|a|^{p}-|a|^{3}}-\frac{|a| \xi}{|a|^{p}-|a|}\right) \frac{\|x\|^{p}}{|a|^{2}\left|a^{2}-1\right|} \quad(\text { for } p>4) .\end{cases}
\end{aligned}
$$

From the above inequalities, we obtain the desired inequality (46).

Corollary 2. Assume that $X$ is a real normed space, $Y$ is a real Banach space, and that $p, \theta, \eta$, and $\xi$ are real constants such that $p \notin\{1,2,3,4\}, 0<|a|<1, \xi>0$, and $\theta>0$. If a mapping $f: X \rightarrow Y$ satisfies $f(0)=0$ and the inequalities in (44) for all $x \in X \backslash\{0\}$, and if $f$ satisfies the inequality (45) 
for all $x_{1}, x_{2}, \ldots, x_{n} \in X \backslash\{0\}$, then there exists a unique mapping $F: X \rightarrow Y$ satisfying (12) for all $x_{1}, x_{2}, \ldots, x_{n} \in X \backslash\{0\}$, the equalities in (13) for all $x \in X$, and (46) for all $x \in X \backslash\{0\}$.

Proof. Replacing $x$ by $\frac{x}{a^{2}}$ and multiplying $\frac{1}{|a|^{6}}$ in the first inequality of (44), we obtain

$$
\left\|\frac{1}{a^{6}} f_{e}(x)-\frac{a^{2}+a^{4}}{a^{6}} f_{e}\left(\frac{x}{a}\right)+f_{e}\left(\frac{x}{a^{2}}\right)\right\| \leq \frac{\eta}{|a|^{6}}\left\|\frac{x}{a^{2}}\right\|^{p}
$$

for all $x \in X \backslash\{0\}$. Analogously, replacing $x$ by $\frac{x}{a^{2}}$ and multiplying $\frac{1}{|a|^{\mid}}$in the second inequality of (44), we have

$$
\left\|\frac{1}{a^{4}} f_{o}(x)-\frac{a+a^{3}}{a^{4}} f_{o}\left(\frac{x}{a}\right)+f_{o}\left(\frac{x}{a^{2}}\right)\right\| \leq \frac{\xi}{|a|^{4}}\left\|\frac{x}{a^{2}}\right\|^{p}
$$

for all $x \in X \backslash\{0\}$.

Let $b=\frac{1}{a}$. Then we obtain the inequalities

$$
\begin{aligned}
& \left\|f_{e}\left(b^{2} x\right)-\left(b^{2}+b^{4}\right) f_{e}(b x)+b^{6} f_{e}(x)\right\| \leq b^{2 p} b^{6} \eta\|x\|^{p}, \\
& \left\|f_{o}\left(b^{2} x\right)-\left(b+b^{3}\right) f_{o}(b x)+b^{4} f_{o}(x)\right\| \leq b^{2 p} b^{4} \xi\|x\|^{p}
\end{aligned}
$$

for all $x \in X \backslash\{0\}$. By Corollary 1, there exists a unique mapping $F: X \rightarrow Y$ satisfying (12) for all $x_{1}, x_{2}, \ldots, x_{n} \in X \backslash\{0\}$, the equalities in (13) for all $x \in X$, and

$$
\begin{aligned}
\|f(x)-F(x)\| & \leq \frac{|b|^{2 p}|b|^{6} \eta\|x\|^{p}}{\left.\left|b^{4}-\right| b\right|^{p}|||b|^{p}-b^{2} \mid}+\frac{|b|^{2 p}|b|^{4} \xi\|x\|^{p}}{\|\left. b\right|^{3}-|b|^{p}|||b|^{p}-|b| \mid} \\
& =\frac{\eta\|x\|^{p}}{\left.\left|a^{4}-\right| a\right|^{p}|||a|^{p}-a^{2} \mid}+\frac{\xi|| x \|^{p}}{\|\left. a\right|^{3}-|a|^{p}|||a|^{p}-|a| \mid}
\end{aligned}
$$

for all $x \in X \backslash\{0\}$.

Author Contributions: Writing-original draft, Y.-H.L. and S.-M.J.; Writing-review \& editing, Y.-H.L. and S.-M.J.

Funding: Soon-Mo Jung was supported by the Basic Science Research Program through the National Research Foundation of Korea (NRF) funded by the Ministry of Education (No. 2016R1D1A1B03931061).

Conflicts of Interest: The authors declare no conflict of interest.

\section{References}

1. Ulam, S.M. A Collection of Mathematical Problems; Interscience Publ.: New York, NY, USA, 1960.

2. Czerwik, S. Functional Equations and Inequalities in Several Variables; World Scientific: London, UK, 2002.

3. Forti, G.L. Hyers-Ulam stability of functional equations in several variables. Aequ. Math. 1995, 50, $143-190$. [CrossRef]

4. Hyers, D.H.; Isac, G.; Rassias, Th.M. Stability of Functional Equations in Several Variables; Birkäuser: Boston, MA, USA, 1998.

5. Jung, S.-M. Hyers-Ulam-Rassias Stability of Functional Equations in Nonlinear Analysis; Springer Optimization and Its Applications; Springer: New York, NY, USA, 2011; Volume 48.

6. Hyers, D.H. On the stability of the linear functional equation. Proc. Natl. Acad. Sci. USA 1941, $27,222-224$. [CrossRef] [PubMed]

7. Brzdęk, J.; Popa, D.; Xu, B. The Hyers-Ulam stability of nonlinear recurrences. J. Math. Anal. Appl. 2007, 335, 443-449. [CrossRef]

8. Hyers, D.H.; Rassias, T.M. Approximate homomorphisms. Aequ. Math. 1992, 44, 125-153. [CrossRef]

9. Găvrută, P. A generalization of the Hyers-Ulam-Rassias stability of approximately additive mappings. J. Math. Anal. Appl. 1994, 184, 431-436. [CrossRef] 
10. Jung, S.-M.; Kim, J.-H. Hyers-Ulam stability of Lagrange's mean value points in two variables. Mathematics 2018, 6, 216. [CrossRef]

11. Jung, S.-M.; Lee, Y.-H. A fixed point approach to the stability of a mean value type functional equation. Mathematics 2017, 5, 78. [CrossRef]

12. Popa, D.; Raşa, I. On the Hyers-Ulam stability of the linear differential equation. J. Math. Anal. Appl. 2011, 381, 530-537. [CrossRef]

13. Qarawani, M.N. Hyers-Ulam stability for Mackey-Glass and Lasota differential equations. J. Math. Res. 2013, 5, 34-40. [CrossRef]

14. Rassias, Th.M. On the stability of the linear mapping in Banach spaces. Proc. Am. Math. Soc. 1978, 72, 297-300. [CrossRef]

15. Brzdęk, J.; Popa, D.; Raşa, I. Hyers-Ulam stability with respect to gauges. J. Math. Anal. Appl. 2017, 453, 620-628. [CrossRef]

16. Lee, Y.-H.; Jung, S.-M.; Rassias, M.T. Uniqueness theorems on functional inequalities concerning cubic-quadratic-additive equation. J. Math. Inequal. 2018, 12, 43-61. [CrossRef]

17. Lee, Y.-H.; Jung, S.-M.; Rassias, M.T. On an n-dimensional mixed type additive and quadratic functional equation. Appl. Math. Comput. 2014, 228, 13-16. [CrossRef]

18. Lee, Y.-H.; Jung, S.-M. A general uniqueness theorem concerning the stability of additive and quadratic functional equations. J. Funct. Spaces 2015. [CrossRef]

19. Lee, Y.-H.; Jung, S.-M. General uniqueness theorem concerning the stability of additive, quadratic, and cubic functional equations. Adv. Differ. Equ. 2016. [CrossRef]

20. Lee, Y.-H.; Jung, S.-M. A fixed point approach to the stability of an additive-quadratic-cubic-quartic type functional equation. J. Funct. Spaces 2016. [CrossRef]

21. Lee, Y.-H.; Jung, S.-M. A general uniqueness theorem concerning the stability of AQCQ type functional equations. Kyungpook Math. J. 2018, 58, 291-305.

(C) 2018 by the authors. Licensee MDPI, Basel, Switzerland. This article is an open access article distributed under the terms and conditions of the Creative Commons Attribution (CC BY) license (http:/ / creativecommons.org/licenses/by/4.0/). 